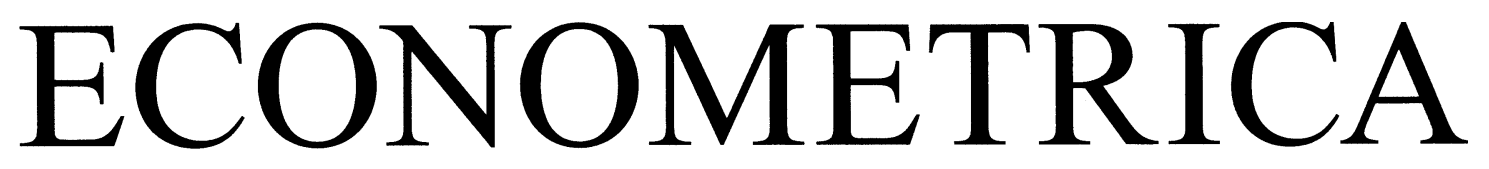

JOURNAL OF THE ECONOMETRIC SOCIETY

An International Society for the Advancement of Economic

Theory in its Relation to Statistics and Mathematics

http://www.econometricsociety.org/

Econometrica, Vol. 85, No. 3 (May, 2017), 959-989

GENERALIZED INSTRUMENTAL VARIABLE MODELS

ANDREW CHESHER

University College London, London, WC1E 6BT, U.K.

ADAM M. ROSEN

Duke University, Durham, NC 27708, U.S.A.

The copyright to this Article is held by the Econometric Society. It may be downloaded, printed and reproduced only for educational or research purposes, including use in course packs. No downloading or copying may be done for any commercial purpose without the explicit permission of the Econometric Society. For such commercial purposes contact the Office of the Econometric Society (contact information may be found at the website http://www.econometricsociety.org or in the back cover of Econometrica). This statement must be included on all copies of this Article that are made available electronically or in any other format. 


\title{
GENERALIZED INSTRUMENTAL VARIABLE MODELS
}

\begin{abstract}
By ANDREW CHESHER AND ADAM M. ROSEN ${ }^{1}$
This paper develops characterizations of identified sets of structures and structural features for complete and incomplete models involving continuous or discrete variables. Multiple values of unobserved variables can be associated with particular combinations of observed variables. This can arise when there are multiple sources of heterogeneity, censored or discrete endogenous variables, or inequality restrictions on functions of observed and unobserved variables. The models generalize the class of incomplete instrumental variable (IV) models in which unobserved variables are singlevalued functions of observed variables. Thus the models are referred to as generalized IV (GIV) models, but there are important cases in which instrumental variable restrictions play no significant role. Building on a definition of observational equivalence for incomplete models the development uses results from random set theory that guarantee that the characterizations deliver sharp bounds, thereby dispensing with the need for case-by-case proofs of sharpness. The use of random sets defined on the space of unobserved variables allows identification analysis under mean and quantile independence restrictions on the distributions of unobserved variables conditional on exogenous variables as well as under a full independence restriction. The results are used to develop sharp bounds on the distribution of valuations in an incomplete model of English auctions, improving on the pointwise bounds available until now. Application of many of the results of the paper requires no familiarity with random set theory.
\end{abstract}

KEYWORDS: Instrumental variables, endogeneity, excess heterogeneity, limited information, partial identification, random sets, incomplete models, English auctions.

\section{INTRODUCTION}

THIS PAPER DEVELOPS CHARACTERIZATIONS OF IDENTIFIED SETS-equivalently sharp bounds-for a wide class of complete and incomplete structural models admitting general forms of unobserved heterogeneity. ${ }^{2}$ To demonstrate the power of these results we apply them to the incomplete model of English auctions introduced in Haile and Tamer (2003) and characterize sharp bounds on the distribution of valuations, tightening the pointwise bounds derived in that paper.

In the models we study multiple values of unobserved variables can be associated with a particular combination of values of observed endogenous and exogenous variables. This occurs in models admitting multiple sources of heterogeneity such as random coefficients, in models with discrete or censored outcomes, and in models in which observed and unobserved variables are constrained by inequality restrictions.

\footnotetext{
${ }^{1}$ We thank the co-editor and three anonymous reviewers for helpful suggestions and feedback that helped to improve the paper. We are grateful to Federico Bugni, Matt Masten, and Francesca Molinari for detailed comments and discussion. We have benefitted from discussion with participants at seminar and conference presentations given at UvA Amsterdam, Boston College, Boston University, Cornell, Chicago, Paris School of Economics, Vanderbilt, the April 2013 Conference on Mathematical Statistics of Partially Identified Objects at Oberwolfach, the 2013 Cowles Summer Econometrics Conference, the 2014 Asia Meeting of the Econometric Society in Taipei, the 2014 China Meeting of the Econometric Society in Xiamen, the Harvard-MIT Econometrics Workshop, Yale, Virginia, Georgetown, Maryland, Johns Hopkins, Toulouse School of Economics, Oxford, Duke, UIUC, Reading, NUS Singapore, and Surrey. Both authors gratefully acknowledge financial support from the UK Economic and Social Research Council through a Grant (Grant RES-589-28-0001) to the ESRC Centre for Microdata Methods and Practice (CeMMAP). Adam Rosen gratefully acknowledges financial support from a British Academy Mid-Career Fellowship, and from the European Research Council (ERC) Grants ERC-2009-StG-240910-ROMETA and ERC-2012-StG-312474. The usual disclaimer applies.

${ }^{2}$ The terms "sharp bounds" and "identified sets" are used interchangeably throughout.
} 
Leading examples of the models we study are classical single equation instrumental variable (IV) models such as the linear model (e.g., Wright (1928), Theil (1953), Basmann (1959)), semiparametric and nonparametric IV models (e.g., Newey and Powell (1989, 2003), and Chernozhukov and Hansen (2005)), and extensions of these models allowing random coefficients and discrete or censored outcomes. In these IV models there are restrictions on the influence of certain exogenous variables on the determination of outcomes and restrictions on the extent of dependence between observed exogenous and unobserved variables. We use the catch-all descriptor generalized instrumental variable (GIV) models to describe the class of models studied in this paper.

The results can also be applied to models in which instrumental variables may play no significant role. The incomplete model of English auctions employed in the leading example is of this type.

Let $Y$ and $Z$ denote, respectively, observed endogenous and exogenous variables, and let $U$ denote unobserved heterogeneity. Lowercase $y, z$, and $u$ denote realizations of these random vectors that may be continuous, discrete, or mixed continuous-discrete. The models studied in this paper place restrictions on a structural function $h(y, z, u)$ mapping the joint support of $Y, Z$, and $U$ onto the real line. The structural function defines the combinations of values of $Y, Z$, and $U$ that may occur through the restriction that $h(Y, Z, U)=0$ almost surely. For example, a classical linear IV model in which $Y_{1}=$ $\alpha Y_{2}+Z \beta+U$ has $h(y, z, u)=y_{1}-\alpha y_{2}-z \beta-u$. More examples are given in Section 2.2.

The prime focus of this paper is on identification of structures. A structure $\left(h, \mathcal{G}_{U \mid Z}\right)$ comprises a structural function $h$ coupled with a family of conditional distributions of $U$ given $Z$,

$$
\mathcal{G}_{U \mid Z} \equiv\left\{G_{U \mid Z}(\cdot \mid z): z \in \mathcal{R}_{Z}\right\},
$$

where $G_{U \mid Z}(\mathcal{S} \mid z)$ is the probability that $U$ belongs to set $\mathcal{S}$ given $Z=z$, and $\mathcal{R}_{Z}$ is the support of exogenous $Z$. Identified sets for structural features, for example, a structural function or some functional of it, are obtained as projections of identified sets of structures.

Level sets of the structural function $h(y, z, u)$ play a central role in the development. Let $\mathcal{R}_{U}$ and $\mathcal{R}_{Y}$ denote the support of $U$ and $Y$, respectively. The random set

$$
\mathcal{U}(Y, Z ; h) \equiv\left\{u \in \mathcal{R}_{U}: h(Y, Z, u)=0\right\}
$$

has realizations $\mathcal{U}(y, z ; h)$ that contain all values of $U$ that can give rise to $Y=y$ when $Z=z$ according to structural function $h$. The random set

$$
\mathcal{Y}(U, Z ; h) \equiv\left\{y \in \mathcal{R}_{Y}: h(y, Z, U)=0\right\}
$$

has realizations $\mathcal{Y}(u, z ; h)$ that contain all values of $Y$ that can occur when $U=u$ and $Z=z$ according to the structural function $h$. Complete models require $\mathcal{Y}(U, Z ; h)$ to be a singleton with probability 1 for all admissible $h$. Incomplete models admit structural functions $h$ such that $\mathcal{Y}(U, Z ; h)$ can have cardinality greater than $1 .^{3}$ Models with multiple sources of heterogeneity, discrete or censored outcomes, or observed and unobserved variables restricted by inequality constraints have sets $\mathcal{U}(Y, Z ; h)$ with realizations that

\footnotetext{
${ }^{3}$ In Chesher and Rosen (2012) we specialize our approach for identification analysis to simultaneous discrete outcome models. There we define incoherent models in which $\mathcal{Y}(U, Z ; h)$ can be empty and we discuss several ways in which incoherence can be addressed, with references to the literature on simultaneous discrete outcome models.
} 
may not be singleton sets. The GIV models studied here require neither of these sets to be singleton and they are generally partially identifying.

This paper provides characterizations of identified sets of structures delivered by GIV models given distributions of observable variables. Previously in this class of models the question of whether sharp bounds are obtained has been primarily handled on a caseby-case basis. The usual approach to proving sharpness is constructive; see, for example, Chesher $(2010,2013)$ and Rosen (2012). This approach requires one to show that every structure in the identified set can deliver the distribution of observed variables. This is often difficult to accomplish and sometimes, as in the auction model of Haile and Tamer (2003), it is infeasible. The methodology introduced here is shown to always deliver characterizations of sharp bounds. It is shown that these sets can be expressed as systems of moment inequalities and equalities to which recently developed inferential procedures are applicable. See, for example, Chesher and Rosen (2013) and Aradillas-Lopez and Rosen (2013) for empirical applications using treatment effect and simultaneous ordered response models.

The results of this paper are obtained using results from random set theory, reviewed in Molchanov (2005) and introduced into econometric identification analysis by Beresteanu, Molchanov, and Molinari (2011), henceforth BMM11. The analysis there employs the random set $\mathcal{Y}(U, Z ; h)$ in models where the identified set can be characterized through a finite number of conditional moment inequalities involving an unobservable and possibly infinite-dimensional nuisance function, such as an equilibrium selection mechanism in econometric models of games. Galichon and Henry (2011) take an alternative approach using optimal transportation theory to characterize sharp parameter bounds in parametrically specified incomplete models.

Instead of using the random $Y$-level set $\mathcal{Y}(U, Z ; h)$, the approach taken in this paper uses the random $U$-level sets $\mathcal{U}(Y, Z ; h)$ to characterize identified sets for $\left(h, \mathcal{G}_{U \mid Z}\right)$ in structural econometric models. The analysis does not require the existence of a representation of the identified set through a finite number of conditional moment equalities involving an unknown nuisance function as required in BMM11. This allows treatment of models with continuous endogenous variables and independence restrictions on the joint distribution of $U$ and $Z$. The analysis here does not require parametric specification for the structural function or the conditional distributions of unobserved heterogeneity as required in Galichon and Henry (2011). The use of random $U$-level sets allows consideration of a variety of restrictions on unobserved heterogeneity common in structural econometrics, including stochastic independence, conditional mean, conditional quantile, and parametric restrictions. Our earlier papers Chesher, Rosen, and Smolinski (2013) and Chesher and Rosen (2012) also used $U$-level sets for identification analysis in models in which outcome variables were required to be discrete, and with $U$ and $Z$ required to be independently distributed. In this paper, using $U$-level sets allows identification analysis in a much broader class of models in which each of the components of endogenous $Y$ can be continuous, discrete, or mixed, and in which the aforementioned alternative restrictions on the joint distribution of $U$ and $Z$ may be imposed.

The main result of this paper is as follows. Let $\theta$ be a structure. This is an object with components that are a structural function $h$ and a collection of conditional distributions $\mathcal{G}_{U \mid Z}$. Let $F_{Y \mid Z}(\cdot \mid z)$ denote a conditional distribution of endogenous variables given $Z=z$. A random set $\mathcal{U}(Y, Z ; h)$ is characterized by the collection of random variables that almost surely lie in the random set. These random variables are called measurable selections of the random set. ${ }^{4}$ It is shown that the identified set of structures delivered by a model

${ }^{4}$ See Definition 1 and Molchanov (2005) for further details. 
given distributions $F_{Y \mid Z}(\cdot \mid z), z \in \mathcal{R}_{Z}$, comprises all $\theta$ admitted by the model such that for almost every $z \in \mathcal{R}_{Z}, G_{U \mid Z}(\cdot \mid z) \in \mathcal{G}_{U \mid Z}$ is the distribution of one of the measurable selections of $\mathcal{U}(Y, Z ; h)$ when $Y$ given $Z=z$ has distribution $F_{Y \mid Z}(\cdot \mid z)$.

Alternative characterizations of this selectionability property deliver alternative characterizations of the identified set. One such alternative is delivered by Artstein's (1983) inequality, characterizing the identified set as those $\theta$ admitted by the model such that the inequality

$$
G_{U \mid Z}(\mathcal{S} \mid z) \geq \mathbb{P}[\mathcal{U}(Y, Z ; h) \subseteq \mathcal{S} \mid Z=z]
$$

holds for all closed sets $\mathcal{S} \subseteq \mathcal{R}_{U}$ and almost every $z \in \mathcal{R}_{Z}$. On the left hand side is the probability that $U$ has a realization in set $\mathcal{S}$ given $Z=z$. On the right hand side is the conditional probability of the occurrence of one of the values of $Y$ such that $\mathcal{U}(Y, Z ; h) \subseteq \mathcal{S}$, under structural function $h$. These are the values of $Y$ that only occur when $U \in \mathcal{S}$. A definition of collections of core determining sets $\mathrm{Q}(h, z)$ is provided such that if (1.3) holds for all sets $\mathcal{S} \in \mathrm{Q}(h, z)$, then it holds for all closed $\mathcal{S} \subseteq \mathcal{R}_{U}$, thereby reducing the collection of inequalities sufficient to characterize the identified set. Conditions are also given under which certain inequalities in (1.3) can be replaced by equalities. ${ }^{5}$ Characterizations employing the Aumann expectation of the random set $\mathcal{U}(Y, Z ; h)$ are also provided.

This result relies on a definition of observational equivalence for models that may admit incomplete structures. This extends the classical definition of observational equivalence for complete models; see, for example, Koopmans (1949), Koopmans and Reiers $\varnothing 1$ (1950), Hurwicz (1950), Rothenberg (1971), Bowden (1973), and Matzkin (2007, 2008). Structures admitted by incomplete models can generate multiple distributions of outcomes; hence our definition of observational equivalence is in terms of random outcome sets $\mathcal{Y}(U, Z ; h)$.

The traction we obtain on characterizing identified sets for structural econometric models relies on a key duality result concerning the random level sets $\mathcal{Y}(U, Z ; h)$ and $\mathcal{U}(Y, Z ; h)$. This allows the development of characterizations of observational equivalence in terms of properties of residual sets $\mathcal{U}(Y, Z ; h)$ rather than outcome sets $\mathcal{Y}(U, Z ; h)$. This is what enables characterization of identified sets in models employing alternative restrictions on the distribution of $U$ and $Z$ such as arise in many structural econometric models.

The results of this paper greatly extend the application of structural models embodying IV exclusion and independence restrictions. IV models with discrete or censored as well as continuous outcomes and with multiple sources of heterogeneity can now be employed. Models that impose inequality restrictions on observed and unobserved variables such as Haile and Tamer's (2003) incomplete model of English auctions are also included in the scope of application as shown in Section 6.

\subsection{Plan}

Section 2 formalizes the GIV model restrictions and provides some leading examples of GIV models. Section 3 provides our generalization of the classical notion of observational equivalence, our duality result, and some accompanying formal set identification characterizations, including a widely applicable construction written in terms of conditional moment inequalities. Section 4 shows how to use the notion of core-determining

\footnotetext{
${ }^{5}$ We extend the use of core determining sets introduced in Galichon and Henry (2011), defining such sets on the support of unobservables instead of the support of endogenous outcomes, and allowing them to depend on the structural function $h$ and the value of exogenous $Z$.
} 
sets to exploit the geometric structure of the random sets $\mathcal{U}(Y, Z ; h)$ to reduce the collection of conditional moment inequalities without losing identifying power. Section 5 shows how restrictions on unobserved heterogeneity and exogenous variables, such as independence, conditional mean, conditional quantile, and parametric restrictions, can be incorporated to further refine characterization of the identified set. Section 6 demonstrates how the application of our results to an incomplete model of English auctions featuring multivariate unobserved heterogeneity delivers a novel and previously unavailable characterization of sharp bounds on the distribution of bidder valuations. Section 7 concludes. All proofs are provided in the Appendix.

\subsection{Notation}

Capital $A$ denotes a point-valued random vector and lowercase letters $a$ denote particular point-valued realizations. For probability measure $\mathbb{P}, \mathbb{P}(\cdot \mid a)$ is used to denote the conditional probability measure given $A=a ; \mathcal{R}_{A_{1} \cdots A_{m}}$ denotes the joint support of random vectors $A_{1}, \ldots, A_{m} ; \mathcal{R}_{A_{1} \mid a_{2}}$ denotes the support of random vector $A_{1}$ conditional on $A_{2}=a_{2} ; q_{A \mid B}(\tau \mid b)$ denotes the $\tau$ conditional quantile of $A$ given $B=b ; \mathrm{F}\left(\mathcal{R}_{A}\right)$ and $\mathrm{K}\left(\mathcal{R}_{A}\right)$ denote the collections of all closed and compact subsets, respectively, of the support of $A$; $A \Perp B$ means that random vectors $A$ and $B$ are stochastically independent; $\varnothing$ denotes the empty set; and $\wedge$ denotes the logical "and" operator. The calligraphic font $(\mathcal{S})$ is reserved for sets, and the sans serif font $(\mathrm{S})$ is reserved for collections of sets. The symbol $\subseteq$ indicates nonstrict set inclusion, $\operatorname{cl}(\mathcal{A})$ denotes the closure of $\mathcal{A}, \partial \mathcal{A}$ denotes the boundary of $\mathcal{A}$, and $C_{h}(\mathcal{S} \mid z)$ denotes the containment functional of random set $\mathcal{U}(Y, Z ; h)$ conditional on $Z=z$, defined in Section 3.2. The notation $F \preccurlyeq \mathcal{A}$ indicates that the distribution $F$ of a random vector is selectionable with respect to the distribution of random set $\mathcal{A}$, and $A \in \operatorname{Sel}(\mathcal{A})$ indicates that random variable $A$ is a measurable selection of random set $\mathcal{A}$, both as defined in Section 3.1. The term $\mathbb{E}[\mathcal{A}]$ refers to the Aumann expectation of $\mathcal{A}$, defined in Section 5.2; $1[\mathcal{E}]$ denotes the indicator function, taking the value 1 if the event $\mathcal{E}$ occurs and 0 otherwise; $\mathbb{R}^{m}$ denotes $m$-dimensional Euclidean space; $\mathbb{R}^{1}$ is abbreviated to $\mathbb{R}$ and for any vector $v \in \mathbb{R}^{m} ;\|v\|$ indicates the Euclidean norm, $\|v\|=\sqrt{v_{1}^{2}+\cdots+v_{m}^{2}}$. So as to deal with sets of measure zero and conditions required to hold almost everywhere, we use the sup and inf operators to denote "essential supremum" and "essential infimum" with respect to the underlying measure when these operators are applied to functions of random variables (e.g., conditional probabilities, expectations, or quantiles). Thus $\sup _{z \in \mathcal{Z}} f(z)$ denotes the smallest value of $c \in \mathbb{R}$ such that $\mathbb{P}[f(Z)>c]=0$ and $\inf _{z \in \mathcal{Z}} f(z)$ denotes the largest value of $c \in \mathbb{R}$ such that $\mathbb{P}[f(Z)<c]=0$.

\section{GIV MODELS}

First there is a formal statement of the restrictions comprising GIV models. Then examples of GIV models are provided.

\subsection{GIV Models}

At various points in the development, the following restrictions are employed.

RESTRICTION A1: The variables $(Y, Z, U)$ are random vectors defined on a probability space $(\Omega, \mathrm{L}, \mathbb{P})$, endowed with the Borel sets on $\Omega$. The support of $(Y, Z, U)$ is a subset of a finite-dimensional Euclidean space. 
RESTRICTION A2: A collection of conditional distributions

$$
\mathcal{F}_{Y \mid Z} \equiv\left\{F_{Y \mid Z}(\cdot \mid z): z \in \mathcal{R}_{Z}\right\}
$$

is identified by the sampling process, where for all $\mathcal{T} \subseteq \mathcal{R}_{Y \mid z}, F_{Y \mid Z}(\mathcal{T} \mid z) \equiv \mathbb{P}[Y \in \mathcal{T} \mid z]$.

RESTRICTION A3: There is an L-measurable function $h(\cdot, \cdot, \cdot): \mathcal{R}_{Y Z U} \rightarrow \mathbb{R}$ such that

$$
\mathbb{P}[h(Y, Z, U)=0]=1,
$$

and there is a collection of conditional distributions

$$
\mathcal{G}_{U \mid Z} \equiv\left\{G_{U \mid Z}(\cdot \mid z): z \in \mathcal{R}_{Z}\right\}
$$

where for all $\mathcal{S} \subseteq \mathcal{R}_{U \mid z}, G_{U \mid Z}(\mathcal{S} \mid z) \equiv \mathbb{P}[U \in \mathcal{S} \mid z]$.

RESTRICTION A4: The pair $\left(h, \mathcal{G}_{U \mid Z}\right)$ belongs to a known set of admissible structures $\mathcal{M}$.

RESTRICTION A5: The random set $\mathcal{U}(Y, Z ; h)$ is closed almost surely $\mathbb{P}[\cdot \mid z]$, each $z \in \mathcal{R}_{Z}$.

RESTRICTION A6: The random set $\mathcal{Y}(Z, U ; h)$ is closed almost surely $\mathbb{P}[\cdot \mid z]$, each $z \in \mathcal{R}_{Z}$.

Restriction A1 defines the probability space on which $(Y, Z, U)$ reside and restricts their support to Euclidean space. ${ }^{6}$ Restriction A2 requires that for each $z \in \mathcal{R}_{Z}, F_{Y \mid Z}(\cdot \mid z)$ is identified. Restriction $\mathrm{A} 3$ posits the existence of structural relation $h$, and provides notation for the collection of conditional distributions $\mathcal{G}_{U \mid Z}$ of $U$ given $Z$.

Restriction A4 imposes model $\mathcal{M}$, the collection of admissible structures $\left(h, \mathcal{G}_{U \mid Z}\right)$. Unlike the previous restrictions, it is refutable based on knowledge of $\mathcal{F}_{Y \mid Z}$ in that it is possible that there is no $\left(h, \mathcal{G}_{U \mid Z}\right) \in \mathcal{M}$ such that $\mathbb{P}[h(Y, Z, U)=0]=1$. In such cases the identified set of structures is empty, indicating model misspecification.

Restrictions A5 and A6 restrict $\mathcal{U}(Y, Z ; h)$ and $\mathcal{Y}(Z, U ; h)$ to be random closed sets. The purpose of these restrictions is to enable use of results from random set theory characterizing the distributions of measurable selections of random closed sets. ${ }^{7}$ These restrictions are satisfied, for example, if $\mathcal{M}$ specifies that all admissible $h$ are continuous in their first and third arguments, respectively, but can also hold more generally. A given econometric model can generally be represented through a variety of different but substantively equivalent structural functions $h$, and judicious choice of this function can often be made to ensure these requirements are satisfied. See Section 2.2 for examples. ${ }^{8}$

\footnotetext{
${ }^{6}$ The restriction of the support of unobserved heterogeneity to a subset of Euclidean space is convenient, but not required for our identification analysis. What is essential for our use of random set theory is that the support of $U$ is a locally compact Hausdorff second countable topological space. Euclidean space fulfills this requirement. For further details we refer to Molchanov (2005).

${ }^{7}$ The definition of a measurable selection of a random set is provided in Section 3.

${ }^{8}$ Importantly, the realizations of $\mathcal{Y}(Z, U ; h)$ and $\mathcal{U}(Y, Z ; h)$ may be unbounded, as closedness merely requires that they contain their limit points. Moreover, whether these sets are closed depends on the underlying topological space. We use the Euclidean topology on $\mathbb{R}^{d}$ throughout, but in some cases other topological spaces could be used to establish closedness. For instance, if $\mathcal{U}(Y, Z ; h)$ can take only a finite number of realizations, then this set is closed in the discrete topology on $\left\{\mathcal{U}(Y, z ; h): z \in \mathcal{R}_{Z}\right\}$; see, for example, Sutherland (2009, p. 94, Exercise 9.1).
} 
Collections of admissible structural functions and families of collections of distributions $\mathcal{G}_{U \mid Z}$ are defined as the following projections of the model $\mathcal{M}$ :

$$
\begin{aligned}
\mathcal{H} & \equiv\left\{h:\left(h, \mathcal{G}_{U \mid Z}\right) \in \mathcal{M} \text { for some } \mathcal{G}_{U \mid Z}\right\}, \\
\mathrm{G}_{U \mid Z} & \equiv\left\{\mathcal{G}_{U \mid Z}:\left(h, \mathcal{G}_{U \mid Z}\right) \in \mathcal{M} \text { for some } h\right\} .
\end{aligned}
$$

A model comprises restrictions on structural functions and on the joint distribution of $(U, Z)$, imposed by specification of admissible structures $\mathcal{M}$ in Restriction A4. Structural functions can be required to belong to a parametric family; there may be semiparametric restrictions, as in index models; there may be a purely nonparametric specification, perhaps with monotonicity or shape restrictions. Admissible collections of conditional distributions $\mathcal{G}_{U \mid Z}$ may be restricted to parametric families but there may be far less restrictive specifications. Many models of interest will impose restrictions on the dependence of $U$ and $Z$ such as mean, quantile, or complete stochastic independence. Our results allow the impact on identification of all these types of restrictions to be studied. Various types of restrictions on $\mathcal{G}_{U \mid Z}$ are considered in Section 5 and the auction model example demonstrates the way in which restrictions are incorporated in structural functions.

The identifying power of a model in the context of a particular process depends not only on the restrictions of the model, but also on the identified joint distribution of observable $(Y, Z)$ delivered by the process. If the distribution of $(Y, Z)$ satisfies certain conditions, then a model may point identify certain structural features. Examples include the rank and completeness conditions that appear when using, respectively, linear simultaneous equations models and nonparametric IV models. In this paper we focus on the characterization of identified sets delivered by general classes of models and we do not consider conditions under which particular models have point identifying power. Consequently there is no consideration here of the impact on identification of alternative forms of the joint distribution of $(Y, Z)$.

\subsection{Examples}

\subsubsection{A Model of English Auctions}

A key application worked through in detail in Section 6 is the incomplete model of English auctions introduced in Haile and Tamer (2003). There are $M$ bidders with continuously and independently distributed valuations with common distribution function $A_{z}(\cdot)$ conditional on auction characteristics $Z=z$. Random variables $V=\left(V_{1}, \ldots, V_{M}\right)$ and $Y \equiv\left(Y_{1}, \ldots, Y_{M}\right)$ denote, respectively, ordered valuations and ordered final bids. ${ }^{9}$ There are restrictions $Y_{m} \leq V_{m}$ for all $m \in\{1, \ldots, M\}$ and $V_{M-1} \leq Y_{M}$, expressing the idea that no person bids more than his/her valuation and no person lets another win at a price he/she is willing to beat.

It is convenient to construct the model in terms of uniform order statistics, $U=$ $\left(U_{1}, \ldots, U_{M}\right)$, that is, ordered identically and independently distributed uniform random variables each with support on $[0,1]$. A structural function that captures the restrictions on bidder behavior is

$$
\begin{aligned}
h(y, z, u)= & \max \left\{u_{M-1}-A_{z}\left(y_{M}\right), 0\right\} \\
& +\sum_{m=1}^{M} \max \left\{A_{z}\left(y_{m}\right)-u_{m}, 0\right\}, \quad y_{1} \leq \cdots \leq y_{M}, u_{1} \leq \cdots \leq u_{M},
\end{aligned}
$$

\footnotetext{
${ }^{9}$ In ordered lists, higher indices indicate larger values and inequalities in random variables hold almost surely.
} 
where $V_{m}=A_{z}^{-1}\left(U_{m}\right)$ has been employed and $A_{z}^{-1}$ is the quantile function associated with the distribution function $A_{z}$. Let $\mathcal{R}_{U}$ denote the orthoscheme of the unit $M$-cube in which $u_{1} \leq u_{2} \leq \cdots \leq u_{M}$. The $U$-level set delivered by the structural function is

$$
\mathcal{U}(y, z ; h)=\left\{u \in \mathcal{R}_{U}:\left(A_{z}\left(y_{M}\right) \geq u_{M-1}\right) \wedge\left(\bigwedge_{m=1}^{M}\left(A_{z}\left(y_{m}\right) \leq u_{m}\right)\right)\right\}
$$

with the $Y$-level set defined similarly. ${ }^{10}$

\subsubsection{Models With Set-Valued Residuals}

An important class of models in the broad class of models covered here are those in which instrumental variable exclusion and independence restrictions play a central role and unobservable variables may be set-valued functions of observed endogenous and exogenous variables. Examples include the binary outcome threshold crossing IV model studied in Chesher (2010) and Chesher and Rosen (2013), the ordered outcome IV model studied in Chesher and Smolinski (2012), and the multiple discrete choice model with endogenous explanatory variables and IV restrictions studied in Chesher, Rosen, and Smolinski (2013). This paper develops results that apply not only in these cases where the outcome variables of interest are discrete, but also to cases with continuous outcomes.

Here are two examples of IV models with set-valued residuals in which outcomes are continuous.

EXAMPLE 1-Random coefficients model with endogenous explanatory variable: A continuous outcome random coefficients model has structural function

$$
h(y, z, u)=y_{1}-z_{1} \gamma-\left(\beta_{2}+u_{2}\right) y_{2}-\left(\beta_{1}+u_{1}\right) .
$$

The random coefficients are $\left(\beta_{1}+U_{1}\right)$ and $\left(\beta_{2}+U_{2}\right)$, with means $\beta_{1}$ and $\beta_{2}$, respectively. The coefficient $\gamma$ multiplying exogenous variables in $h$ could also be random. The level sets are

$$
\begin{aligned}
& \mathcal{Y}(u, z ; h)=\left\{\left(z_{1} \gamma+\left(\beta_{2}+u_{2}\right) y_{2}+\left(\beta_{1}+u_{1}\right), y_{2}\right): y_{2} \in \mathcal{R}_{Y_{2}}\right\}, \\
& \mathcal{U}(y, z ; h)=\left\{u \in \mathcal{R}_{U}: u_{1}=y_{1}-z_{1} \gamma-\beta_{1}-\beta_{2} y_{2}-u_{2} y_{2}\right\} .
\end{aligned}
$$

This is an incomplete limited information single equation instrumental variable model, in contrast to the complete simultaneous equations random coefficient model studied by Masten (2014), in which were established conditions for point identification of the marginal distributions of the random coefficients.

EXAMPLE 2-Interval censored endogenous explanatory variables: This model extends one of the cases studied in Manski and Tamer (2002) in which an exogenous explanatory variable is censored to situations in which the censored explanatory variable is endogenous. Let $g(\cdot, \cdot, \cdot): \mathbb{R} \times \mathbb{R}^{k} \times \mathbb{R} \rightarrow \mathbb{R}$ be increasing in its first argument and strictly increasing and continuous in its third argument such that

$$
Y_{1}=g\left(Y_{2}^{*}, Z_{1}, U\right)
$$

\footnotetext{
${ }^{10}$ For $\mathcal{Y}(u, z ; h)$ replace $u \in \mathcal{R}_{U}$ with $y \in \mathcal{R}_{Y}$ in (2.1), where $\mathcal{R}_{Y}$ is the support of the distribution of ordered final bids.
} 
where endogenous variable $Y_{2}^{*} \in \mathbb{R}$ is interval censored with $\mathbb{P}\left[Y_{2 l} \leq Y_{2}^{*} \leq Y_{2 u}\right]=1$ for observed variables $Y_{2 l}, Y_{2 u}$. No further restriction is placed on the process determining the realizations of $Y_{2 l}, Y_{2 u}$. The structural function is

$$
h(y, z, u)=\max \left\{y_{1}-g\left(y_{2 u}, z_{1}, u\right), 0\right\}+\max \left\{g\left(y_{2 l}, z_{1}, u\right)-y_{1}, 0\right\},
$$

with $y \equiv\left(y_{1}, y_{2 l}, y_{2 u}\right)$ and $y_{2 l} \leq y_{2 u}$. The resulting level sets are

$$
\begin{aligned}
& \mathcal{Y}(u, z ; h)=\left\{y \in \mathcal{R}_{Y}: g\left(y_{2 l}, z_{1}, u\right) \leq y_{1} \leq g\left(y_{2 u}, z_{1}, u\right) \wedge y_{2 l} \leq y_{2 u}\right\}, \\
& \mathcal{U}(y, z ; h)=\left[g^{-1}\left(y_{2 u}, z_{1}, y_{1}\right), g^{-1}\left(y_{2 l}, z_{1}, y_{1}\right)\right]
\end{aligned}
$$

where the function $g^{-1}(\cdot, \cdot, \cdot)$ is the inverse of $g(\cdot, \cdot, \cdot)$ with respect to its third argument, so that for all $y_{2}, z_{1}$, and $u, g^{-1}\left(y_{2}, z_{1}, g\left(y_{2}, z_{1}, u\right)\right)=u$.

Characterizations of identified sets for both these models are given in the working paper Chesher and Rosen (2015), where there are also examples of a model with endogenous censoring and a model with endogenous variables measured with error. The methods of this paper also apply to simultaneous equations and triangular models; see, for instance, Chesher and Rosen (2012) for some examples involving incomplete models with discrete endogenous variables.

\section{OBSERVATIONAL EQUIVALENCE}

\subsection{Observational Equivalence and Selectionability in Outcome Space}

The conventional definition of observational equivalence found in the econometrics literature applies when each structure, $m \in \mathcal{M}$, delivers a single collection of conditional distributions,

$$
\mathcal{P}_{Y \mid Z}(m) \equiv\left\{P_{Y \mid Z}(\cdot \mid z ; m): z \in \mathcal{R}_{Z}\right\}
$$

where $P_{Y \mid Z}(\cdot \mid z ; m)$ is the conditional distribution of $Y$ given $Z=z$ delivered by structure $m .{ }^{11}$ In such complete models structures $m$ and $m^{\prime}$ are observationally equivalent if $\mathcal{P}_{Y \mid Z}(m)=\mathcal{P}_{Y \mid Z}\left(m^{\prime}\right)$ almost surely.

Structures admitted by incomplete models may generate more than one collection of conditional distributions. Let $\mathrm{P}_{Y \mid Z}(m)$ denote the set of collections of conditional distributions that can be generated by a structure $m$. Considering two structures $m$ and $m^{\prime}$ there may be a collection of conditional distributions $\mathcal{F}_{Y \mid Z}^{*}$ that lies in both $\mathrm{P}_{Y \mid Z}(m)$ and $\mathrm{P}_{Y \mid Z}\left(m^{\prime}\right)$ and a collection $\mathcal{F}_{Y \mid Z}^{* *}$ that lies in only one of the collections $\mathrm{P}_{Y \mid Z}(m)$ and $\mathrm{P}_{Y \mid Z}\left(m^{\prime}\right)$. Structures $m$ and $m^{\prime}$ are observationally equivalent in identification analysis employing $\mathcal{F}_{Y \mid Z}^{*}$ but not in identification analysis employing $\mathcal{F}_{Y \mid Z}^{* *}$.

When we work with incomplete models, as in this paper, observational equivalence is defined with respect to the collection of distributions $\mathcal{F}_{Y \mid Z}$ under consideration. ${ }^{12}$ The

\footnotetext{
${ }^{11}$ See, for example, Koopmans and Reiersøl (1950), Hurwicz (1950), Rothenberg (1971), Bowden (1973), and Matzkin (2007, 2008).

${ }^{12}$ In our formulation of observational equivalence and characterizations of identified sets, we continue to work with conditional distributions of endogenous and latent variables, $F_{Y \mid Z}(\cdot \mid z)$ and $G_{U \mid Z}(\cdot \mid z)$, respectively, for almost every $z \in \mathcal{R}_{Z}$. Knowledge of the distribution of $Z$ combined with $F_{Y \mid Z}(\cdot \mid z)$ or $G_{U \mid Z}(\cdot \mid z)$ a.e. $z \in \mathcal{R}_{Z}$ is equivalent to knowledge of the joint distribution of $(Y, Z)$, denoted $F_{Y Z}$, or that of $(U, Z)$, denoted $G_{U Z}$, respectively. It is formally shown in Appendix B of Chesher and Rosen (2015) that our characterizations using selectionability conditional on $Z=z$, almost everywhere (a.e.) $z \in \mathcal{R}_{Z}$, are equivalent to using analogous selectionability criteria for the joint distributions $F_{Y Z}$ or $G_{U Z}$.
} 
definition of observational equivalence uses random set theory constructs, specifically measurable selections of a random set and selectionability of probability distributions with respect to the distribution of a random set, which are defined now. ${ }^{13}$

DEFINITION 1: Let $W$ and $\mathcal{W}$ denote a random vector and random set defined on the same probability space. The random vector $W$ is a measurable selection of $\mathcal{W}$, denoted $W \in \operatorname{Sel}(\mathcal{W})$, if $W \in \mathcal{W}$ with probability 1 . The distribution $F_{W}$ of random vector $W$ is selectionable with respect to the distribution of random set $\mathcal{W}$, abbreviated $F_{W} \preccurlyeq \mathcal{W}$, if there exists a random vector $\tilde{W}$ distributed $F_{W}$ and a random set $\tilde{\mathcal{W}}$ with the same distribution as $\mathcal{W}$ such that $\tilde{W} \in \operatorname{Sel}(\tilde{\mathcal{W}})$.

In the context of this paper a structure $\left(h, \mathcal{G}_{U \mid Z}\right)$ induces a distribution for the random outcome set $\mathcal{Y}(U, Z ; h)$ conditional on $Z=z$, for all $z \in \mathcal{R}_{Z}$. The distributions of $Y$ given $Z=z$ that are selectionable with respect to the distribution of $\mathcal{Y}(U, Z ; h)$ given $Z=z$ for almost every $z \in \mathcal{R}_{Z}$ are precisely those distributions for which $h(Y, Z, U)=0$ can hold with probability 1 for the given structure $\left(h, \mathcal{G}_{U \mid Z}\right)$. This leads to the following definitions. $^{14}$

DEFINITION 2: Under Restrictions A1-A3, two structures $\left(h, \mathcal{G}_{U \mid Z}\right)$ and $\left(h^{\prime}, \mathcal{G}_{U \mid Z}^{\prime}\right)$ are observationally equivalent with respect to a given collection of conditional distributions $\left\{F_{Y \mid Z}(\cdot \mid z): z \in \mathcal{R}_{Z}\right\}$, if $F_{Y \mid Z}(\cdot \mid z) \preccurlyeq \mathcal{Y}(U, z ; h)$ when $U \sim G_{U \mid Z}(\cdot \mid z)$ and $F_{Y \mid Z}(\cdot \mid z) \preccurlyeq$ $\mathcal{Y}\left(U, z ; h^{\prime}\right)$ when $U \sim G_{U \mid Z}^{\prime}(\cdot \mid z)$ for almost every $z \in \mathcal{R}_{Z}$.

DEFINITION 3: Under Restrictions A1-A4, the identified set of structures $\left(h, \mathcal{G}_{U \mid Z}\right)$ with respect to the collection of distributions $\mathcal{F}_{Y \mid Z}$ are those admissible structures such that the conditional distributions $F_{Y \mid Z}(\cdot \mid z) \in \mathcal{F}_{Y \mid Z}$ are selectionable with respect to the conditional distributions of random set $\mathcal{Y}(U, z ; h)$ when $U \sim G_{U \mid Z}(\cdot \mid z)$, a.e. $z \in \mathcal{R}_{Z}$ :

$$
\mathcal{M}^{*} \equiv\left\{\left(h, \mathcal{G}_{U \mid Z}\right) \in \mathcal{M}: F_{Y \mid Z}(\cdot \mid z) \preccurlyeq \mathcal{Y}(U, z ; h) \text { when } U \sim G_{U \mid Z}(\cdot \mid z) \text {, a.e. } z \in \mathcal{R}_{Z}\right\} \text {. }
$$

A definition of set identification of structural features follows directly from Definition 3. Any functional, $\psi\left(h, \mathcal{G}_{U \mid Z}\right)$, of a structure $\left(h, \mathcal{G}_{U \mid Z}\right)$ is a structural feature and its identified set is a projection of the set $\mathcal{M}^{*} .^{15}$

DEFINITION 4: The identified set of structural features $\psi(\cdot, \cdot)$ under Restrictions A1-A4 is the projection of $\mathcal{M}^{*}$ :

$$
\Psi \equiv\left\{\psi\left(h, \mathcal{G}_{U \mid Z}\right):\left(h, \mathcal{G}_{U \mid Z}\right) \in \mathcal{M}^{*}\right\}
$$

The structural features of interest depend on the circumstances encountered. In this paper the focus is on the identified set of structures $\mathcal{M}^{*}$ from which identified sets of structural features are obtained by projection.

\footnotetext{
${ }^{13}$ See Molchanov (2005). These definitions are Definition 2.2 on page 26 and Definition 2.19 on page 34 .

${ }^{14}$ The identified set $\mathcal{M}^{*}$ in Definition 3 depends on the collection of conditional distributions $\mathcal{F}_{Y \mid Z}$, although we do not make this dependence explicit in our notation.

${ }^{15}$ The identified set of structural features $\Psi$ depends on both $\mathcal{M}$ and the conditional distributions $\mathcal{F}_{Y \mid Z}$, but for ease of notation we suppress this dependence.
} 


\subsection{Observational Equivalence and Selectionability in U-Space}

In this section we set out an equivalent, and in many cases more useful, characterization of observational equivalence in terms of (a) random sets $\mathcal{U}(Y, Z ; h)$ whose distribution is determined by the structural function, $h$, and a collection of conditional distributions of outcomes $\mathcal{F}_{Y \mid Z}$ and (b) selectionability of the distributions of unobservables, $\mathcal{G}_{U \mid Z}$, with respect to the distributions of these random $U$-level sets. This alternative characterization follows directly from a simple duality property of the two types of level sets of structural functions, namely that for all $h$ and $z$,

$$
u^{*} \in \mathcal{U}\left(y^{*}, z ; h\right) \Longleftrightarrow y^{*} \in \mathcal{Y}\left(u^{*}, z ; h\right),
$$

which is so because each inclusion holds if and only if $h\left(y^{*}, z, u^{*}\right)=0$.

The advantage of this new characterization is that it allows direct imposition of restrictions on the collection of distributions $\mathcal{G}_{U \mid Z}$ admitted by the model $\mathcal{M}$. Such restrictionsfor example, mean, quantile, full independence, and parametric restrictions-are the bread and butter of econometrics. The characterization is set out in the following two theorems.

THEOREM 1: Let Restrictions A1-A3 hold. Then for any $z \in \mathcal{R}_{Z}, F_{Y \mid Z}(\cdot \mid z)$ is selectionable with respect to the conditional distribution of $\mathcal{Y}(U, Z ; h)$ given $Z=z$ when $U \sim G_{U \mid Z}(\cdot \mid z)$ if and only if $G_{U \mid Z}(\cdot \mid z)$ is selectionable with respect to the conditional distribution of $\mathcal{U}(Y, Z ; h)$ given $Z=z$ when $Y \sim F_{Y \mid Z}(\cdot \mid z)$.

THEOREM 2: Let Restrictions A1-A3 hold. Then (i) structures $\left(h, \mathcal{G}_{U \mid Z}\right)$ and $\left(h^{*}, \mathcal{G}_{U \mid Z}^{*}\right)$ are observationally equivalent with respect to $\mathcal{F}_{Y \mid Z}$ if and only if $G_{U \mid Z}(\cdot \mid z)$ and $G_{U \mid Z}^{*}(\cdot \mid z)$ are selectionable with respect to the conditional (on $Z=z$ ) distributions of random sets $\mathcal{U}(Y, Z ; h)$ and $\mathcal{U}\left(Y, Z ; h^{*}\right)$, respectively, a.e. $z \in \mathcal{R}_{Z}$, where the conditional distributions of these random sets are delivered by the collection of distributions $\mathcal{F}_{Y \mid Z}$, and (ii) if additionally Restriction A4 holds, then the identified set of structures $\left(h, \mathcal{G}_{U \mid Z}\right)$ are those elements of $\mathcal{M}$ such that $G_{U \mid Z}(\cdot \mid z)$ is selectionable with respect to the conditional (on $Z=z$ ) distribution of $\mathcal{U}(Y, Z ; h)$ a.e. $z \in \mathcal{R}_{Z}$, where the conditional distributions of $\mathcal{U}(Y, Z ; h)$ are delivered by $\mathcal{F}_{Y \mid Z}$.

Theorem 2 expresses observational equivalence and the characterization of the identified set of structures $\left(h, \mathcal{G}_{U \mid Z}\right)$ in terms of selectionability of members of $\mathcal{G}_{U \mid Z}$ relative to the conditional distribution of the random residual set $\mathcal{U}(Y, Z ; h)$. Any characterization of the selectionability condition in Theorem 2 delivers a characterization of the identified set.

One such characterization that applies when $\mathcal{U}(Y, Z ; h)$ is a random closed set, given in Corollary 1, uses Artstein's inequality characterization of selectionability; see, for example, Artstein (1983), Norberg (1992), and Molchanov (2005, Section 1.4.8). This employs the conditional containment functional of $\mathcal{U}(Y, Z ; h)$, defined as

$$
C_{h}(\mathcal{S} \mid z) \equiv \mathbb{P}[\mathcal{U}(Y, Z ; h) \subseteq \mathcal{S} \mid z] .
$$

COROLLARY 1: Under Restrictions A1-A5, the identified set can be written

$$
\mathcal{M}^{*} \equiv\left\{\left(h, \mathcal{G}_{U \mid Z}\right) \in \mathcal{M}: \forall \mathcal{S} \in \mathrm{F}\left(\mathcal{R}_{U}\right), C_{h}(\mathcal{S} \mid z) \leq G_{U \mid Z}(\mathcal{S} \mid z) \text {, a.e. } z \in \mathcal{R}_{Z}\right\},
$$

where $\mathrm{F}\left(\mathcal{R}_{U}\right)$ denotes the collection of all closed subsets of $\mathcal{R}_{U}$. 
There are inequalities in this characterization for almost every value of the instrument $z \in \mathcal{R}_{Z}$ and for all closed subsets of $\mathcal{R}_{U}$. So the results in the next section showing how the collection of sets of values of $U$ defining the characterization of $\mathcal{M}^{*}$ can be reduced are crucial.

\section{CORE-DETERMINING TEST SETS}

A collection $\mathrm{Q}(h, z)$ of core-determining test sets $\mathcal{S}$ is characterized for any $h$ and any $z \in \mathcal{R}_{Z}$, such that if, for all $\mathcal{S}$ in $\mathrm{Q}(h, z)$,

$$
C_{h}(\mathcal{S} \mid z) \leq G_{U \mid Z}(\mathcal{S} \mid z),
$$

then the same inequality holds for all closed sets $\mathcal{S} \subseteq \mathcal{R}_{U} \cdot{ }^{16}$ Consequently, the characterization of $\mathcal{M}^{*}$ in (3.2) can be written with $\mathrm{Q}(h, z)$ replacing $\mathrm{F}\left(\mathcal{R}_{U}\right) .{ }^{17}$ The following definitions are employed.

DEFINITION 5: The conditional support of random set $\mathcal{U}(Y, Z ; h)$ given $Z=z$ is $\mathrm{U}(h, z)$ :

$$
\mathrm{U}(h, z) \equiv\left\{\mathcal{U} \subseteq \mathcal{R}_{U}: \exists y \in \mathcal{R}_{Y \mid z} \text { such that } \mathcal{U}=\mathcal{U}(y, z ; h)\right\} .
$$

The collection of sets $\mathrm{U}^{*}(h, z)$ comprises all sets that are unions of elements of $\mathrm{U}(h, z)$,

$$
\mathrm{U}^{*}(h, z) \equiv\left\{\mathcal{U} \subseteq \mathcal{R}_{U}: \exists \mathcal{Y} \subseteq \mathcal{R}_{Y \mid z} \text { such that } \mathcal{U}=\mathcal{U}(\mathcal{Y}, z ; h)\right\},
$$

where

$$
\mathcal{U}(\mathcal{Y}, z ; h) \equiv \bigcup_{y \in \mathcal{Y}} \mathcal{U}(y, z ; h)
$$

For any $\mathcal{S} \subseteq \mathcal{R}_{U}, h$, and $z$ define the subcollection $\mathrm{U}^{\mathcal{S}}(h, z)$ of $\mathrm{U}(h, z)$ as

$$
\mathrm{U}^{\mathcal{S}}(h, z) \equiv\{\mathcal{U} \in \mathrm{U}(h, z): \mathcal{U} \subseteq \mathcal{S}\} .
$$

Lemma 1 establishes that, for any $\left(h, \mathcal{G}_{U \mid Z}\right)$ and $z$, if the inequality (4.1) holds for all sets $\mathcal{S}$ in the collection of unions $\mathrm{U}^{*}(h, z)$, then it holds for all $\mathcal{S} \subseteq \mathcal{R}_{U}$. Theorem 3 defines a collection of core-determining test sets $\mathrm{Q}(h, z)$, which is a refinement of $\mathrm{U}^{*}(h, z)$.

LEMMA 1: Let Restrictions A1-A3 hold. Let $z \in \mathcal{R}_{Z}, h \in \mathcal{H}$, and $\mathcal{S} \subseteq \mathcal{R}_{U}$. Let $\mathcal{U}_{\mathcal{S}}(h, z)$ denote the union of all sets in $\mathrm{U}^{\mathcal{S}}(h, z)$ :

$$
\mathcal{U}_{\mathcal{S}}(h, z) \equiv \bigcup_{\mathcal{U} \in \cup^{\mathcal{S}}(h, z)} \mathcal{U}
$$

If $C_{h}\left(\mathcal{U}_{\mathcal{S}}(h, z) \mid z\right) \leq G_{U \mid Z}\left(\mathcal{U}_{\mathcal{S}}(h, z) \mid z\right)$, then $C_{h}(\mathcal{S} \mid z) \leq G_{U \mid Z}(\mathcal{S} \mid z)$.

\footnotetext{
${ }^{16}$ Core-determining sets may also be dependent on $G_{U \mid Z}(\cdot \mid z)$ as set out in Theorem 3 below, but this is not made explicit in the notation.

${ }^{17}$ Galichon and Henry (2011) introduced core-determining sets for identification analysis considering sets in outcome space and characterizing core-determining sets for incomplete models that satisfy a certain monotonicity requirement. Here no monotonicity condition is imposed and their definition is extended by introducing core-determining sets for the characterizations in $U$-space developed in Section 3, and by allowing core-determining sets to be specific to the structural relation $h$ and covariate value $z$.
} 
THEOREM 3: Let Restrictions A1-A3 hold. For any $(h, z) \in \mathcal{H} \times \mathcal{R}_{Z}$, let $\mathrm{Q}(h, z) \subseteq$ $\mathrm{U}^{*}(h, z)$, such that for any $\mathcal{S} \in \mathrm{U}^{*}(h, z)$ with $\mathcal{S} \notin \mathrm{Q}(h, z)$, there exist nonempty collections $\mathrm{S}_{1}, \mathrm{~S}_{2} \subseteq \mathrm{U}^{\mathcal{S}}(h, z)$ with $\mathrm{S}_{1} \cup \mathrm{S}_{2}=\mathrm{U}^{\mathcal{S}}(h, z)$ such that

$$
\mathcal{S}_{1} \equiv \bigcup_{\mathcal{T} \in \mathrm{S}_{1}} \mathcal{T}, \quad \mathcal{S}_{2} \equiv \bigcup_{\mathcal{T} \in \mathcal{S}_{2}} \mathcal{T}, \quad \text { and } \quad G_{U \mid Z}\left(\mathcal{S}_{1} \cap \mathcal{S}_{2} \mid z\right)=0
$$

with $\mathcal{S}_{1}, \mathcal{S}_{2} \in \mathrm{Q}(h, z)$. Then $C_{h}(\mathcal{S} \mid z) \leq G_{U \mid Z}(\mathcal{S} \mid z)$ for all $\mathcal{S} \in \mathrm{Q}(h, z)$ implies that $C_{h}(\mathcal{S} \mid z) \leq$ $G_{U \mid Z}(\mathcal{S} \mid z)$ holds for all closed $\mathcal{S} \subseteq \mathcal{R}_{U}$, so that the collection of sets $\mathrm{Q}(h, z)$ is coredetermining.

This theorem implies, for example, that if $h$ is such that all level sets $\mathcal{U}(y, z ; h)$ are connected, then sets $\mathcal{S}$ that can only be written as unions of disjoint sets in $\mathrm{U}(h, z)$ can be excluded from consideration. Corollary 2 gives cases in which certain containment functional inequalities can be replaced by equalities.

COROLLARY 2: Let $\partial \mathcal{S}$ denote the boundary of set $\mathcal{S}$. Define

$$
\begin{aligned}
\mathrm{Q}^{E}(h, z) \equiv & \left\{\mathcal{S} \in \mathrm{Q}(h, z): G_{U \mid Z}(\partial \mathcal{S} \mid z)=0\right. \text { and } \\
& \left.\forall y \in \mathcal{R}_{Y} \text { either } \mathcal{U}(y, z ; h) \subseteq \mathcal{S} \text { or } \mathcal{U}(y, z ; h) \subseteq \operatorname{cl}\left(\mathcal{S}^{c}\right)\right\} .
\end{aligned}
$$

Under the conditions of Theorem 3, the collection of equalities and inequalities

$$
\begin{array}{ll}
C_{h}(\mathcal{S} \mid z)=G_{U \mid Z}(\mathcal{S} \mid z), & \text { all } \mathcal{S} \in \mathrm{Q}^{E}(h, z), \\
C_{h}(\mathcal{S} \mid z) \leq G_{U \mid Z}(\mathcal{S} \mid z), & \text { all } \mathcal{S} \in \mathrm{Q}^{I}(h, z) \equiv \mathrm{Q}(h, z) \backslash \mathrm{Q}^{E}(h, z)
\end{array}
$$

holds if and only if $C_{h}(\mathcal{S} \mid z) \leq G_{U \mid Z}(\mathcal{S} \mid z)$ for all $\mathcal{S} \in \mathrm{Q}(h, z)$.

There are two classes of models in which all members of $\mathrm{Q}(h, z)$ belong to $\mathrm{Q}^{E}(h, z)$, so that the identified set may be characterized by only conditional moment equalities.

- Models where $\mathcal{U}(Y, Z ; h)$ is a singleton set with probability 1 . This includes models with an additive unobservable as in the classical linear IV model, the nonparametric IV model of Newey and Powell (2003), and the quantile IV model of Chernozhukov and Hansen (2005).

- Complete models for which $\mathcal{Y}(U, Z ; h)$ is a singleton set with probability 1 . In such models for any $z$ and any $y \neq y^{\prime}$ the sets $\mathcal{U}(y, z ; h)$ and $\mathcal{U}\left(y^{\prime}, z ; h\right)$ have measure zero intersection. Since $\mathrm{Q}(h, z)$ is a collection of sets that are unions of sets on the support of $\mathcal{U}(Y, Z ; h)$, for all $(y, z)$, any $h$, and all $\mathcal{S} \in \mathrm{Q}(h, z)$, either $\mathcal{U}(y, z ; h) \subseteq \mathcal{S}$ or $\mathcal{U}(y, z ; h) \subseteq$ $\operatorname{cl}\left(\mathcal{S}^{c}\right)$.

In models in which all endogenous variables are discrete with finite support, the collection of core-determining sets is finite and it is possible to enumerate them all. An algorithm for doing this is provided in Chesher and Rosen (2012). In models with continuous endogenous variables such as the auction model, there is typically an uncountable infinity of core-determining sets and in applications a selection must be made. This is discussed further in Sections 6 and $7 .{ }^{18}$

\footnotetext{
${ }^{18}$ The working paper Chesher and Rosen (2015) additionally explains how particular selections are made in numerical illustrations of identified sets for Examples 1 and 2 in Section 2.2 in which endogenous variables are continuous.
} 


\section{IDENTIFIED SETS UNDER RESTRICTIONS ON THE DISTRIBUTION OF $(U, Z)$}

Theorem 2 provides a characterization of the structures $\left(h, \mathcal{G}_{U \mid Z}\right)$ contained in the identified set delivered by a model $\mathcal{M}$ and a collection of distributions $\mathcal{F}_{Y \mid Z}$. A key element of econometric models is restrictions on the conditional distributions of unobserved variables. In this section we show how some commonly employed restrictions on admissible collections of conditional distributions $\mathcal{G}_{U \mid Z}$ refine the characterization of an identified set. The restrictions considered are full stochastic independence, conditional mean, and conditional quantile independence.

\subsection{Stochastic Independence}

RESTRICTION SI-Stochastic Independence: For all collections $\mathcal{G}_{U \mid Z}$ of conditional distributions admitted by $\mathcal{M}, U$ and $Z$ are stochastically independent.

Under Restriction SI conditional distributions $G_{U \mid Z}(\cdot \mid z)$ cannot vary with $z$ and we write $G_{U}$ in place of the collection $\mathcal{G}_{U \mid Z}$, where for all $z, G_{U}(\cdot)=G_{U \mid Z}(\cdot \mid z)$.

It follows from Theorem 2 that a structure $\left(h, G_{U}\right) \in \mathcal{M}$ belongs to $\mathcal{M}^{*}$ if and only if $G_{U}$ is selectionable with respect to the conditional distribution of the random set $\mathcal{U}(Y, Z ; h)$ induced by $F_{Y \mid Z}(\cdot \mid z)$ a.e. $z \in \mathcal{R}_{Z}$. Four characterizations of such structures are set out in Theorem 4.

THEOREM 4: Let Restrictions A1-A5 and SI hold. Then

$$
\begin{aligned}
\mathcal{M}^{*} & =\left\{\left(h, G_{U}\right) \in \mathcal{M}: G_{U}(\cdot) \preccurlyeq \mathcal{U}(Y, z ; h) \text { when } Y \sim F_{Y \mid Z}(\cdot \mid z), \text { a.e. } z \in \mathcal{R}_{Z}\right\} \\
& =\left\{\begin{array}{c}
\left(h, G_{U}\right) \in \mathcal{M}: \forall \mathcal{S}_{I} \in \mathrm{Q}^{I}(h, z), \forall \mathcal{S}_{E} \in \mathrm{Q}^{E}(h, z), \\
\left.C_{h}\left(\mathcal{S}_{I} \mid z\right) \leq G_{U}\left(\mathcal{S}_{I}\right), C_{h}\left(\mathcal{S}_{E} \mid z\right)=G_{U}\left(\mathcal{S}_{E}\right), \text { a.e. } z \in \mathcal{R}_{Z}\right\} .
\end{array}\right.
\end{aligned}
$$

If Restriction $\mathrm{A} 6$ also holds, then, equivalently,

$$
\begin{aligned}
& \mathcal{M}^{*}=\left\{\left(h, G_{U}\right) \in \mathcal{M}: F_{Y \mid Z}(\cdot \mid z) \preccurlyeq \mathcal{Y}(U, z ; h) \text { when } U \sim G_{U}(\cdot), \text { a.e. } z \in \mathcal{R}_{Z}\right\}, \\
&=\left\{\left(h, G_{U}\right) \in \mathcal{M}: \forall \mathcal{K} \in \mathrm{K}\left(\mathcal{R}_{Y}\right),\right. \\
&\left.F_{Y \mid Z}(\mathcal{K} \mid z) \leq G_{U}\{\mathcal{Y}(U, z ; h) \cap \mathcal{K} \neq \emptyset\}, \text { a.e. } z \in \mathcal{R}_{Z}\right\},
\end{aligned}
$$

where $\mathrm{K}\left(\mathcal{R}_{Y}\right)$ denotes the collection of compact sets in $\mathcal{R}_{Y}$.

Theorem 4 presents alternative representations of the identified set under Restriction SI. Characterizations (5.3) and (5.1) arise directly on application of the restriction to Definition 3.1 and Theorem 2, respectively. The characterization (5.2) applies Theorem 3 and Corollary 2 to define the identified set in terms of the conditional containment functional of the random $\operatorname{set} \mathcal{U}(Y, Z ; h)$. This representation employs core-determining sets to reduce the number of moment conditions in the characterization, and distinguishes which ones hold as equalities and which hold as inequalities.

The characterization (5.4) defines the identified set through conditional moment inequalities involving the capacity functional of $\mathcal{Y}(U, Z ; h)$. This delivers the characterizations provided in Appendix D.2 of BMM11 and in Galichon and Henry (2011) when applied to incomplete models of games. In general this characterization using random sets in $Y$-space, $\mathcal{R}_{Y}$, requires the inequalities to hold for all compact sets $\mathcal{K} \subset \mathcal{R}_{Y}$. Simplification is sometimes possible: Galichon and Henry (2011) provides core-determining 
sets in $\mathcal{R}_{Y}$ when a certain monotonicity condition holds; BMM11 Appendix D.3 provides alternative conditions under which some inequalities are redundant.

In many cases, the representation (5.2) will be the simplest to use. This characterization uses the containment functional of the random set $\mathcal{U}(Y, Z ; h)$, which has support in $U$ space. This allows the use of core-determining sets on $\mathcal{R}_{U}$ given by Theorem 3 , which is in general a smaller collection of sets than all compact sets in $Y$-space. The ability to exploit the structure of sets $\mathcal{U}(Y, Z ; h)$ for this purpose is a benefit of working in the space of unobserved heterogeneity. Our construction is based on core-determining sets specific to each $(h, z)$ pair, while the collections of core-determining sets working in $Y$-space characterized by Galichon and Henry (2011) under monotonicity are not.

A further difference between characterizations (5.2) and (5.4) is the way in which they incorporate restrictions on the distribution of unobserved heterogeneity. Given an admissible distribution $G_{U}$, use of characterization (5.4) requires computation of the probability that $\mathcal{Y}(U, z ; h)$ hits $\mathcal{K}$ for each compact set $\mathcal{K}$. This has typically been achieved by means of simulation from each conjectured distribution $G_{U}$; see, for example, Appendix D.2 of BMM11 and Henry, Meango, and Queyranne (2015).

The characterization (5.2) in $U$-space shows that there is an alternative to simulating draws from the distribution of unobservables. Computation using (5.2) requires computation of $G_{U}(\mathcal{S})$ for each conjectured distribution $G_{U}$ and each core-determining set $\mathcal{S}$, which can be done either by simulation or by numerical integration. Regarding $\mathbb{P}[\mathcal{U}(Y, Z ; h) \subseteq \mathcal{S} \mid z]$, there is the equivalence

$$
\mathbb{P}[\mathcal{U}(Y, Z ; h) \subseteq \mathcal{S} \mid z]=\mathbb{P}[Y \in \mathcal{A}(\mathcal{S}, Z ; h) \mid z],
$$

where

$$
\mathcal{A}(\mathcal{S}, Z ; h) \equiv\left\{y \in \mathcal{R}_{Y}: \mathcal{U}(y, Z ; h) \subseteq \mathcal{S}\right\}
$$

is the set of values of $y$ that can occur only if $\mathcal{U}(y, Z ; h) \subseteq \mathcal{S}$. Thus $\mathbb{P}[\mathcal{U}(Y, Z ; h) \subseteq \mathcal{S} \mid z]$ is the probability of an event concerning the observed variables $(Y, Z)$, which, for any structural function $h$, is point-identified and can be computed directly as a function of $F_{Y \mid Z}(\cdot \mid z)$. For instance, an analog estimator using the empirical distribution of $(Y, Z)$ based on $n$ observations with discrete $Z$ is the sample average

$$
\hat{P}_{n}[\mathcal{U}(Y, Z ; h) \subseteq \mathcal{S} \mid z] \equiv \frac{1}{n_{z}} \sum_{i=1}^{n} 1\left[y_{i} \in \mathcal{A}(\mathcal{S}, z ; h) \wedge z_{i}=z\right]
$$

with $n_{z}=\sum_{i=1}^{n} 1\left[z_{i}=z\right]$. With continuous $Z$, a kernel estimator could be used for $\hat{P}_{n}[\mathcal{U}(Y, Z ; h) \subseteq \mathcal{S} \mid z]$. The particular form of this probability depends of course on the structural functions, $h$, admitted by the model under consideration. ${ }^{19}$

The characterization of identified sets employing random sets in $U$-space leads directly to Corollary 3, which characterizes bounds on the structural function $h$ under Restriction SI, without explicit reference to $G_{U}$.

\footnotetext{
${ }^{19}$ For explicit examples of the form of $\mathbb{P}[\mathcal{U}(Y, Z ; h) \subseteq \mathcal{S} \mid z]$ in terms of events involving observable variables, see expressions (6.6), (6.7), and (6.8) in the analysis of the auction model in Section 6, and also the containment functional inequalities in Section 6.2 and Supplementary Appendix C of the working paper version (Chesher and Rosen (2015)).
} 
COROLLARY 3: If Restrictions $\mathrm{A} 1-\mathrm{A} 5$ and $\mathrm{SI}$ hold, and $\mathcal{G}_{U \mid Z}$ is otherwise unrestricted, the set

$$
\overline{\mathcal{H}^{*}}=\left\{h \in \mathcal{H}: \forall \mathcal{S} \in \mathrm{S}, \sup _{z \in \mathcal{R}_{Z}} C_{h}(\mathcal{S} \mid z) \leq \inf _{z \in \mathcal{R}_{Z}}\left(1-C_{h}\left(\mathcal{S}^{c} \mid z\right)\right)\right\}
$$

comprises bounds on $h$, such that $\mathcal{H}^{*} \subseteq \overline{\mathcal{H}^{*}}$ for any collection of test sets $\mathrm{S}$. If, in addition, $G_{U}(\partial \mathcal{S})=0$, then (5.5) may be strengthened to $\sup _{z \in \mathcal{R}_{Z}} C_{h}(\mathrm{cl}(\mathcal{S}) \mid z) \leq \inf _{z \in \mathcal{R}_{Z}}(1-$ $\left.C_{h}\left(\operatorname{cl}\left(\mathcal{S}^{c}\right) \mid z\right)\right)$.

In (5.5), $1-C_{h}\left(\mathcal{S}^{c} \mid z\right)=\mathbb{P}[\mathcal{U}(Y, Z ; h) \cap \mathcal{S} \neq \emptyset \mid Z=z]$ is the conditional capacity functional of $\mathcal{U}(Y, Z ; h)$ given $Z=z$. The result is obtained using an upper bound on $G_{U}(\mathcal{S})$ produced by applying the containment functional inequalities in Theorem 4 to $\mathcal{S}^{c}$, the complement of $\mathcal{S}$, in conjunction with the containment functional inequality (4.1). This simple projection result is useful in situations in which $G_{U}$ is not parametrically specified, characterizing bounds on the structural function without the need to explicitly posit distributions of unobserved $U$ that, coupled with these structural functions, could have delivered the data. The condition $G_{U}(\partial \mathcal{S})=0$ holds in many instances, for example, when sets $\mathcal{S}$ are convex and $G_{U}$ is restricted to be absolutely continuous with respect to Lebesgue measure by Theorem 1 of Lang (1986). The continuity restriction rules out, for example, the possibility of unobservable random variables having point mass at threshold values in discrete outcome models, in which case the strengthened version of inequalities can be tighter.

\subsection{Mean Independence}

RESTRICTION MI-Mean Independence: Let $G_{U \mid Z}$ comprise all collections $\mathcal{G}_{U \mid Z}$ of conditional distributions for $U$ given $Z$ satisfying $E[U \mid z]=c$, a.e. $z \in \mathcal{R}_{Z}$, for some fixed, finite $c$ belonging to a known set $\mathcal{C} \subseteq \mathcal{R}_{U}$.

This restriction limits the collection $\mathcal{G}_{U \mid Z}$ to those containing conditional distributions $G_{U \mid Z}(\cdot \mid z)$ such that $E[U \mid z]$ is equal to a constant $c$ that does not vary with $z$. This covers cases where numerical values are provided for some components of $c$ but not for others. For instance, in a model with bivariate $U$, Restriction MI with $\mathcal{C}=\left\{\left(c_{1}, c_{2}\right): c_{1}=0, c_{2} \in \mathbb{R}\right\}$ restricts $E\left[U_{1} \mid z\right]=0$, which could be a normalization, and restricts $E\left[U_{2} \mid z\right]$ to be invariant with respect to $z$.

Under Restriction MI it is convenient to characterize the selectionability criterion of Theorem 2 using the Aumann expectation. ${ }^{20}$

DEFINITION 6: The Aumann expectation of random set $\mathcal{A}$ is

$$
\mathbb{E}[\mathcal{A}] \equiv \operatorname{cl}\{E[A]: A \in \operatorname{Sel}(\mathcal{A}) \text { and } E[A]<\infty\}
$$

Molchanov (2005, p. 151). The Aumann expectation of random set $\mathcal{A}$ conditional on $B=$ $b$ is

$$
\mathbb{E}[\mathcal{A} \mid b] \equiv \operatorname{cl}\{E[A \mid b]: A \in \operatorname{Sel}(\mathcal{A}) \text { and } E[A \mid b]<\infty\}
$$

\footnotetext{
${ }^{20}$ For random sets defined on finite-dimensional spaces, such as those considered under Restriction A3, application of the closure operator in Definition 6 is not necessary; see, for example, Nguyen (2006, p. 184).
} 
A characterization of the identified sets for structural function $h$ and for the structure $\left(h, \mathcal{G}_{U \mid Z}\right)$ under Restriction MI is given in the following theorem.

THEOREM 5: Let Restrictions $\mathrm{A} 1-\mathrm{A} 5$ and $\mathrm{MI}$ hold and suppose that $(\Omega, \mathrm{L}, \mathbb{P})$ is nonatomic. Then the identified set for structural function $h$ comprises those functions $h$ such that some $c \in \mathcal{C}$ is an element of the Aumann expectation of $\mathcal{U}(Y, Z ; h)$ conditional on $Z=z$ a.e. $z \in \mathcal{R}_{Z}$ :

$$
\mathcal{H}^{*}=\left\{h \in \mathcal{H}: \exists c \in \mathcal{C} \text { s.t. for almost every } z \in \mathcal{R}_{Z}, c \in \mathbb{E}[\mathcal{U}(Y, Z ; h) \mid z]\right\} .
$$

The identified set for $\left(h, \mathcal{G}_{U \mid Z}\right)$ is

$$
\begin{aligned}
\mathcal{M}^{*}= & \left\{\left(h, \mathcal{G}_{U \mid Z}\right) \in \mathcal{M}: h \in \mathcal{H}^{*} \text { and } G_{U \mid Z}(\cdot \mid z) \preccurlyeq \mathcal{U}(Y, Z ; h)\right. \\
& \text { conditional on } \left.Z=z, \text { a.e. } z \in \mathcal{R}_{Z}\right\},
\end{aligned}
$$

where by virtue of Restriction $M I$ all structures $\left(h, \mathcal{G}_{U \mid Z}\right) \in \mathcal{M}^{*} \subseteq \mathcal{M}$ are such that for some $c \in \mathcal{C}, E[U \mid z]=$ c a.e. $z \in \mathcal{R}_{Z}$.

Knowledge of properties of the random set $\mathcal{U}(Y, Z ; h)$ can be helpful in characterizing its Aumann expectation and, consequently, in determining whether any particular $h$ is in $\mathcal{H}^{*}$. For example, if $\mathcal{U}(Y, Z ; h)$ is integrable, then from Molchanov (2005, Theorem 2.1.47-iv, p. 171), $c \in \mathbb{E}[\mathcal{U}(Y, Z ; h) \mid z]$ if and only if

$$
\inf _{v \in \mathcal{R}_{U}:\|v\|=1}\left\{E[m(v, \mathcal{U}(Y, Z ; h)) \mid z]-v^{\prime} c\right\} \geq 0
$$

where for any set $\mathcal{S}$,

$$
m(v, \mathcal{S}) \equiv \sup \{v \cdot s: s \in \mathcal{S}\}
$$

denotes the support function of $\mathcal{S}$ evaluated at $v \cdot{ }^{21}$ BMM11 employed Theorem 2.1.47-iv of Molchanov (2005, p. 171) when using the conditional Aumann expectation of random outcome set $\mathcal{Y}(Z, U ; h)$ to characterize its measurable selections. As was the case in their analysis, use of the support function inequality (5.6) can provide computational tractability. This is particularly so when the dimension of $\mathcal{R}_{U}$ exceeds 1 , as it can be used to circumvent explicit computation of the Aumann expectation for the purpose of verifying whether $c \in \mathbb{E}[\mathcal{U}(Y, Z ; h) \mid z]$ for some $c \in \mathcal{C}$.

In comparison to the sharp bound characterizations of BMM11, if the structural function $h$ is additively separable in $Y$, the two representations are equivalent, differing only by a trivial location shift. On the other hand, if $h$ is not additively separable in $U$, the conditional mean restriction MI cannot generally be written as a conditional mean restriction on $Y$, and previous identification results using the Aumann expectation in $Y$-space appear inapplicable. Theorem 5 provides a characterization for the identified set in such cases. The insight of BMM11 that the support function can be used to bypass explicit computation of the Aumann expectation of a multivariate random set remains applicable.

Theorem 5 can be generalized to characterize $\mathcal{H}^{*}$ under more general forms of conditional mean restrictions as expressed in Restriction $\mathrm{MI}^{*}$.

\footnotetext{
${ }^{21} \mathrm{~A}$ random set is integrable if it has at least one measurable selection with finite $\mathbf{L}^{1}$ norm; see Molchanov (2005, Definition 1.1, p. 146, and Definition 1.11(ii) pp. 150-151).
} 
RESTRICTION MI*: Let $\mathrm{G}_{U \mid Z}$ comprise all collections $\mathcal{G}_{U \mid Z}$ of conditional distributions for $U$ given $Z$ such that for some known function $d(\cdot, \cdot): \mathcal{R}_{U} \times \mathcal{R}_{Z} \rightarrow \mathbb{R}^{k_{d}}$, $E[d(U, Z) \mid z]=c$ a.e. $z \in \mathcal{R}_{Z}$, for some fixed $c$ belonging to a known set $\mathcal{C} \subseteq \mathbb{R}^{k_{d}}$, where $d(u, z)$ is continuous in $u$ for all $z \in \mathcal{R}_{Z}$.

Restriction $\mathrm{MI}^{*}$ requires that the conditional mean given $Z=z$ of some function $d(U, Z)$ taking values in $\mathbb{R}^{k_{d}}$ does not vary with respect to $z$. This restriction can accommodate models that impose conditional mean restrictions on functions of unobservables $U$, for example, homoskedasticity restrictions or restrictions on covariances of elements of $U$. To express the identified set delivered under restriction $\mathrm{MI}^{*}$ define

$$
\mathcal{D}(y, z ; h) \equiv\{d(u, z): u \in \mathcal{U}(y, z ; h)\} .
$$

Then $\mathcal{D}(Y, Z ; h)$ is a random set of feasible values for $d(U, Z)$ given observed $(Y, Z)$. This set is closed under the requirement of Restriction MI* that $d(\cdot, z)$ is continuous for each $z$. The arguments that deliver Theorem 5 yield the following result.

COROLlaRY 4: Let Restrictions A1-A5 and MI* hold and suppose that $(\Omega, \mathrm{L}, \mathbb{P})$ is nonatomic. Then the identified set for structural function $h$ are those $h$ such that there exists at least one $c \in \mathcal{C}$ that is an element of the Aumann expectation of $\mathcal{D}(Y, Z ; h)$ conditional on $Z=z$ a.e. $z \in \mathcal{R}_{Z}$ :

$$
\mathcal{H}^{*}=\left\{h \in \mathcal{H}: \exists c \in \mathcal{C} \text { s.t. for almost every } z \in \mathcal{R}_{Z}, c \in \mathbb{E}[\mathcal{D}(Y, Z ; h) \mid z]\right\} .
$$

The identified set for $\left(h, \mathcal{G}_{U \mid Z}\right)$ is

$$
\begin{aligned}
\mathcal{M}^{*}= & \left\{\left(h, \mathcal{G}_{U \mid Z}\right) \in \mathcal{M}: h \in \mathcal{H}^{*} \text { and } G_{U \mid Z}(\cdot \mid z) \preccurlyeq \mathcal{U}(Y, Z ; h)\right. \\
& \text { conditional on } \left.Z=z \text {, a.e. } z \in \mathcal{R}_{Z}\right\},
\end{aligned}
$$

where, by Restriction $\mathrm{MI}^{*}$, all structures $\left(h, \mathcal{G}_{U \mid Z}\right) \in \mathcal{M}^{*} \subseteq \mathcal{M}$ are such that for some $c \in \mathcal{C}$, $E[d(U, Z) \mid z]=$ c a.e. $z \in \mathcal{R}_{Z}$.

As before, the support function can be useful for computation because it can be used to verify whether $c \in \mathbb{E}[\mathcal{D}(Y, Z ; h) \mid z]$ by use of (5.6) with $\mathcal{D}(Y, Z ; h)$ in place of $\mathcal{U}(Y, Z ; h)$ as long as $\mathcal{D}(Y, Z ; h)$ is integrable.

\subsection{Quantile Independence}

Conditional quantile restrictions on the distribution of unobserved $U$ can also be accommodated. This is illustrated in a simple setting under Restriction IS.

RESTRICTION IS-Interval Support: Let $\mathcal{R}_{U} \subseteq \mathbb{R}$ and for all $(y, z) \in \mathcal{R}_{Y Z}$,

$$
\mathcal{U}(y, z ; h)=[\underline{u}(y, z ; h), \bar{u}(y, z ; h)],
$$

where possibly $\underline{u}(y, z ; h)=-\infty$ or $\bar{u}(y, z ; h)=+\infty$, in which case the corresponding endpoint of the interval (5.7) is open.

The conditional quantile restriction is as follows. 
RESTRICTION QI-Quantile Independence: For some known $\tau \in(0,1)$ and some known set $\mathcal{C} \subseteq \mathbb{R}, \mathrm{G}_{U \mid Z}$ comprises all collections $\mathcal{G}_{U \mid Z}$ of conditional distributions for $U$ given $Z$ that satisfy $q_{U \mid Z}(\tau \mid z)=c$, a.e. $z \in \mathcal{R}_{Z}$ for some $c \in \mathcal{C}$.

THEOREM 6: Let Restrictions A1-A5, IS, and QI hold. Then (i) the identified set for structural function $h$ is

$$
\begin{aligned}
\mathcal{H}^{*}= & \{h \in \mathcal{H}: \exists c \in \mathcal{C} \text { s.t. } \\
& \left.\sup _{z \in \mathcal{R}_{Z}} \mathbb{P}[\bar{u}(Y, Z ; h)<c \mid z] \leq \tau \leq \inf _{z \in \mathcal{R}_{Z}} \mathbb{P}[\underline{u}(Y, Z ; h) \leq c \mid z]\right\} .
\end{aligned}
$$

(ii) If $\underline{u}(Y, Z ; h)$ and $\bar{u}(Y, Z ; h)$ are continuously distributed conditional on $Z=z$, a.e. $z \in \mathcal{R}_{Z}$, then an equivalent formulation of $\mathcal{H}^{*}$ is given by

$$
\mathcal{H}^{*}=\left\{h \in \mathcal{H}: \exists c \in \mathcal{C} \text { s.t. } \sup _{z \in \mathcal{R}_{Z}} \underline{q}(\tau, z ; h) \leq c \leq \inf _{z \in \mathcal{R}_{Z}} \bar{q}(\tau, z ; h)\right\},
$$

where $\underline{q}(\tau, z ; h)$ and $\bar{q}(\tau, z ; h)$ are the $\tau$-quantiles of, respectively, $\underline{u}(Y, Z ; h)$ and $\bar{u}(Y$, $Z ; h)$.

(iii) The identified set for $\left(h, \mathcal{G}_{U \mid Z}\right)$ is

$$
\begin{aligned}
\mathcal{M}^{*}= & \left\{\left(h, \mathcal{G}_{U \mid Z}\right) \in \mathcal{M}: h \in \mathcal{H}^{*} \text { and } G_{U \mid Z}(\cdot \mid z) \preccurlyeq \mathcal{U}(Y, Z ; h)\right. \\
& \text { conditional on } \left.Z=z, \text { a.e. } z \in \mathcal{R}_{Z}\right\},
\end{aligned}
$$

where following from Restriction $\mathrm{QI}$, all structures $\left(h, \mathcal{G}_{U \mid Z}\right) \in \mathcal{M}^{*} \subseteq \mathcal{M}$ are such that for some $c \in \mathcal{C}, q_{U \mid Z}(\tau \mid z)=c$, a.e. $z \in \mathcal{R}_{Z}$.

Under Restriction QI both $G_{U \mid Z}((-\infty, c] \mid z) \geq \tau$ and $G_{U \mid Z}((-\infty, c) \mid z) \leq \tau$ hold, and the inequalities comprising (5.8) then follow from $u(Y, Z ; h) \leq U \leq \bar{u}(Y, Z ; h)$ and use of the conditional containment inequality $C_{h}(\mathcal{S} \mid z) \leq G_{U \mid Z}(\mathcal{S} \mid z)$ applied to test sets $\mathcal{S}=(-\infty, c)$ and $\mathcal{S}=(c, \infty)$. In the proof of Theorem 6 it is shown that for any $h$ and any $c$, if the containment functional inequalities hold for these two test sets, then a random variable $\tilde{U}$ can be found with admissible conditional distributions $\mathcal{G}_{U \mid Z}$ such that the containment functional inequalities hold for all closed test sets in $\mathcal{R}_{U}$. From Corollary 1 it follows that the characterization (5.8) is sharp.

The second part of Theorem 6 follows because when $\underline{u}(Y, Z ; h)$ and $\bar{u}(Y, Z ; h)$ are continuous, the lower bound in (5.8) is equivalent to $\mathbb{P}[\overline{\bar{u}}(Y, Z ; h) \leq c \mid z]$, and both inequalities in (5.8) involving cumulative distributions of $\underline{u}(Y, Z ; h)$ and $\bar{u}(Y, Z ; h)$ may be inverted. Then $\mathcal{H}^{*}$ may be equivalently expressed as inequalities involving the lower and upper envelopes, $q(\tau, z ; h)$ and $\bar{q}(\tau, z ; h)$, respectively, of conditional quantile functions for measurable selections of $\mathcal{U}(Y, Z ; h)$. The third part of Theorem 6 states that the identified set of structures $\left(h, \mathcal{G}_{U \mid Z}\right)$ are elements of $\mathcal{H}^{*}$ paired with distributions $G_{U \mid Z}(\cdot \mid z)$ that are selectionable with respect to the conditional distribution of $\mathcal{U}(Y, Z ; h)$ given $Z=z$, a.e. $z \in \mathcal{R}_{Z}$.

These results can be applied, for instance, to single equation regression models with censored variables and quantile restrictions, such as those of Hong and Tamer (2003), and Khan, Ponomareva, and Tamer (2011), as well as the model of Example 2 in Section 2.2 in which the endogenous variable is censored. ${ }^{22}$

\footnotetext{
${ }^{22}$ See Appendix C of the working paper Chesher and Rosen (2015) for the characterization delivered by models with structural function as described in Example 2.
} 


\section{AN INCOMPLETE MODEL OF AUCTIONS}

This section demonstrates the power of our approach in delivering characterizations of identified sets by resolving the question posed in Haile and Tamer (2003) (henceforth HT) regarding the sharpness of the bounds on valuation distributions in English auctions developed in that paper. Our new characterization of the identified set includes the pointwise lower and upper bounds on valuation distribution functions derived in HT and refines these bounds with additional inequalities that restrict the shape of the valuation distribution function as it passes between the HT bounds. Our approach obviates the need for a constructive proof of sharpness, which, as noted in HT, is difficult to produce in the auction model.

We study the HT model in which a known number, $M$, of bidders have valuations that are independent realizations drawn from a common conditional distribution of valuations given observed auction characteristics $Z=z$, denoted $A_{z}(v) \equiv \mathbb{P}[V \leq v \mid Z=z]$. Bidders engage in an open outcry ascending English auction. HT develops pointwise bounds on $A_{z}(v)$ that hold at each value $v$. To simplify the exposition there is no minimum reserve price or minimum bid increment. ${ }^{23}$

We first set the auction model in the framework introduced in Section 2, defining a structural function for the auction model and its $U$-level sets. We then apply Theorem 4 and the results in Section 4 on core-determining sets to deliver a characterization of sharp bounds on valuation distributions supported by the HT model.

As in HT we consider the information contained in the joint distribution of ordered final bids, $Y=\left(Y_{1}, \ldots, Y_{M}\right)$. Here and later, in $M$-element ordered lists, index $M$ identifies the highest value. Realizations of $V=\left(V_{1}, \ldots, V_{M}\right)$ are ordered, continuously distributed valuations of the bidders. Let $\tilde{U} \in[0,1]^{M}$ be $M$ mutually independent uniform random variables with $\tilde{U} \Perp Z$ and with order statistics $U \equiv\left(U_{1}, \ldots, U_{M}\right)$. Ordered valuations can be expressed as functions of these uniform order statistics as $V_{m}=A_{z}^{-1}\left(U_{m}\right), m \in\{1, \ldots, M\}$, where $A_{z}^{-1}$ is the quantile function associated with the distribution function $A_{z}$.

The HT model includes the restrictions that (i) no one bids more than their valuation, which implies that the inequality in order statistics $V_{m} \geq Y_{m}$ holds for all $m$, and (ii) no one allows an opponent to win at a price they are willing to beat, which requires the second highest valuation to be no larger than the winning bid, $Y_{M} \geq V_{M-1} \cdot{ }^{24,25}$ Applying the strictly monotone function $A_{z}$ to both sides of these inequalities gives

$$
A_{z}\left(Y_{M}\right) \geq U_{M-1} \quad \forall m: A_{z}\left(Y_{m}\right) \leq U_{m},
$$

which leads to a structural function for the HT model:

$$
\begin{aligned}
h(y, z, u)= & \max \left\{u_{M-1}-A_{z}\left(y_{M}\right), 0\right\} \\
& +\sum_{m=1}^{M} \max \left\{A_{z}\left(y_{m}\right)-u_{m}, 0\right\}, \quad y_{1} \leq \cdots \leq y_{M}, u_{1} \leq \cdots \leq u_{M} .
\end{aligned}
$$

The vector of $M$ uniform order statistics, $U$, has constant density function equal to $M$ ! supported on $\mathcal{R}_{U}$, which is the orthoscheme of the unit $M$-cube in which $U_{1} \leq \cdots \leq U_{M}$;

\footnotetext{
${ }^{23}$ With a known reserve price $r$ our analysis delivers the identified set for the distribution of valuations truncated below at $r$. A simple adjustment to accommodate a nonzero minimum bid increment is indicated below.

${ }^{24} \mathrm{As}$ before, inequalities in random variables are required to hold almost surely.

${ }^{25}$ With a minimum bid increment of $\Delta$ this inequality is $Y_{M}+\Delta \geq V_{M-1}$.
} 
see David and Nagaraja (2003). Let $G_{U}(\mathcal{S})$ denote the probability mass placed by this distribution on a set $\mathcal{S} \subseteq \mathcal{R}_{U}$. Structures in this model are pairs $\left(h, G_{U}\right)$, where $h$ is the structural function given in (6.1) and $G_{U}$ is the known distribution of uniform order statistics. The $U$-level sets of the structural function (6.1) are

$$
\mathcal{U}(y, z ; h)=\left\{u \in \mathcal{R}_{U}:\left(A_{z}\left(y_{M}\right) \geq u_{M-1}\right) \wedge \bigwedge_{m=1}^{M}\left(A_{z}\left(y_{m}\right) \leq u_{m}\right)\right\} .
$$

Lemma 1 states that core-determining test sets that characterize the identified set for $A_{z}(\cdot)$ are unions of these $U$-level sets. There is an uncountable infinity of such unions, so we study a selection of unions of $U$-level sets, $\mathcal{S}\left(y^{\prime}, y_{M}^{\prime \prime}, z ; h\right)$, defined as

$$
\mathcal{S}\left(y^{\prime}, y_{M}^{\prime \prime}, z ; h\right) \equiv \bigcup_{y_{M} \in\left[y_{M}^{\prime}, y_{M}^{\prime \prime}\right]} \mathcal{U}\left(\left(y_{1}^{\prime}, y_{2}^{\prime}, \ldots, y_{M-1}^{\prime}, y_{M}\right), z ; h\right), \quad y_{M}^{\prime \prime} \geq y_{M}^{\prime} \geq \cdots \geq y_{1}^{\prime} .
$$

The part of $\mathcal{R}_{U}$ occupied by such a contiguous union is

$$
\mathcal{S}\left(y^{\prime}, y_{M}^{\prime \prime}, z ; h\right)=\left\{u:\left(A_{z}\left(y_{M}^{\prime \prime}\right) \geq u_{M-1}\right) \wedge\left(\bigwedge_{m=1}^{M}\left(u_{m} \geq A_{z}\left(y_{m}^{\prime}\right)\right)\right)\right\} .
$$

These are termed contiguous unions because they are unions of $U$-sets that are contiguous along a sequence determined by an interval of values $\left[y_{M}^{\prime}, y_{M}^{\prime \prime}\right]$ of the largest ordered bid. Contiguous unions of $U$-level sets and unions of such contiguous unions comprise a coredetermining collection of sets.

Applied to contiguous unions the containment functional inequality given in (5.2) in Theorem 4 requires that for each $z$, all valuation distributions, $A_{z}$, in the identified set satisfy

$$
G_{U}\left(\mathcal{S}\left(y^{\prime}, y_{M}^{\prime \prime}, z ; h\right)\right) \geq \mathbb{P}\left[\mathcal{U}(Y, Z ; h) \subseteq \mathcal{S}\left(y^{\prime}, y_{M}^{\prime \prime}, z ; h\right) \mid z\right]
$$

for all $M$, and $y^{\prime}$ and $y_{M}^{\prime \prime}$ such that $y_{M}^{\prime \prime} \geq y_{M}^{\prime} \geq \cdots \geq y_{1}^{\prime}$. If a model restricts the valuation distribution to a parametric family, these inequalities place restrictions on parameter values.

On the left hand side of (6.5) there is the probability

$$
\begin{aligned}
& G_{U}\left(\mathcal{S}\left(y^{\prime}, y_{M}^{\prime \prime}, z ; h\right)\right) \\
& \quad=M ! \int_{A_{z}\left(y_{M}^{\prime}\right)}^{1} \int_{A_{z}\left(y_{M-1}^{\prime}\right)}^{\min \left(u_{M}, A_{z}\left(y_{M}^{\prime \prime}\right)\right)} \int_{A_{z}\left(y_{M-2}^{\prime}\right)}^{u_{M-1}} \cdots \int_{A_{z}\left(y_{1}^{\prime}\right)}^{u_{2}} d u_{M} d u_{M-1} d u_{M-2} \cdots d u_{1},
\end{aligned}
$$

which follows directly from (6.4) and the known uniform distribution of the uniform order statistics $U$ on the orthoscheme $\mathcal{R}_{U}$. This is a degree- $M$ polynomial function of the conditional distribution function of valuations evaluated at $y_{M}^{\prime \prime} \geq y_{M}^{\prime} \geq \cdots \geq y_{1}^{\prime}$, which may be, but need not be, $M+1$ distinct values. It does not depend on the probability distribution of final bids and in estimation of the identified set it can be calculated without reference to data. 
On the right hand side of (6.5) is the containment functional of $\mathcal{S}\left(y^{\prime}, y_{M}^{\prime \prime}, z ; h\right):^{26}$

$$
\mathbb{P}\left[\mathcal{U}(Y, Z ; h) \subseteq \mathcal{S}\left(y^{\prime}, y_{M}^{\prime \prime}, z ; h\right) \mid z\right]=\mathbb{P}\left[\left(y_{M}^{\prime \prime} \geq Y_{M} \geq y_{M}^{\prime}\right) \wedge\left(\bigwedge_{m=1}^{M-1}\left(Y_{m} \geq y_{m}^{\prime}\right)\right) \mid z\right] .
$$

This does not depend on the distribution $A_{z}$. It is entirely determined by the joint distribution of ordered bids and in estimation of the identified set it can be calculated once and for all at the start of the search for the identified set of valuation distributions.

Particular choices of $y^{\prime}$ and $y_{M}^{\prime \prime}$ deliver the pointwise bounds in HT as follows. Plugging $y_{M}^{\prime \prime}=+\infty, y_{m}^{\prime}=-\infty$ for $m<n$, and $y_{m}^{\prime}=v$ for $m \geq n$ into (6.5) delivers the inequality

$$
\mathbb{P}\left[U_{n} \geq A_{z}(v) \mid Z=z\right] \geq \mathbb{P}\left[Y_{n} \geq v \mid z\right] .
$$

The marginal distribution of $U_{n}$, the $n$th of $M$ uniform order statistics, is $\operatorname{Beta}(n, M+1-$ $n)$. Let $Q(\cdot, n, M)$ denote the quantile function of this distribution. Transforming both sides of (6.7) expressed in terms of distribution functions using this quantile function gives

$$
A_{z}(v) \leq Q\left(\mathbb{P}\left[Y_{n} \leq v \mid z\right], n, M\right),
$$

which holds for all $n$, leading to

$$
A_{z}(v) \leq \inf _{n \in\{1, \ldots, M\}} Q\left(\mathbb{P}\left[Y_{n} \leq v \mid z\right], n, M\right),
$$

which is the pointwise upper bound in Theorem 1 of HT.

Plugging $y_{M}^{\prime \prime}=v$ and $y^{\prime}=(-\infty, \ldots,-\infty)$ into (6.5) delivers, after a similar manipulation, the inequality

$$
A_{z}(v) \geq Q\left(\mathbb{P}\left[Y_{M} \leq v \mid z\right], M-1, M\right),
$$

which is the pointwise lower bound in Theorem 3 of HT.

These $M+1$ contiguous unions are the only sets in the list of core-determining sets that are determined by a single value of $Y$. Choices of $y^{\prime}$ and $y_{M}^{\prime \prime}$ determined by more than one value of $Y$ lead to inequalities that restrict values of $A_{z}(\cdot)$ at more than one value of its argument. These inequalities restrict the path taken by the distribution function $A_{z}(\cdot)$ as it passes between the HT pointwise bounds. For example, plugging $y_{M}^{\prime \prime}=v_{3}$ and $y^{\prime}=\left(-\infty, \ldots,-\infty, v_{1}, v_{2}\right)$ into (6.5) delivers the inequality

$$
\begin{aligned}
& M\left(1-A_{z}\left(v_{3}\right)\right)\left(A_{z}\left(v_{3}\right)^{M-1}-A_{z}\left(v_{1}\right)^{M-1}\right) \\
& \quad-M A_{z}\left(v_{1}\right)^{M-1}\left(A_{z}\left(v_{3}\right)-A_{z}\left(v_{2}\right)\right)+\left(A_{z}\left(v_{3}\right)^{M}-A_{z}\left(v_{2}\right)^{M}\right) \\
& \quad \geq \mathbb{P}\left[v_{3} \geq Y_{M} \geq v_{2} \wedge Y_{M-1} \geq v_{1} \mid z\right],
\end{aligned}
$$

which, for any $z$, holds for all valuation distributions $A_{z}$ in the identified set for all $v_{1} \leq$ $v_{2} \leq v_{3}$.

To demonstrate that the new inequalities can be informative we consider a population of two bidder auctions in which the valuation distribution is an equally weighted mixture of normal distributions, $N(10,1)$ and $N\left(12.5,0.5^{2}\right)$, truncated below at zero. The final

\footnotetext{
${ }^{26}$ The inequalities in $Y$ on the right hand side follow directly from inspection of (6.2) and (6.4) once they are written in terms of $A_{z}(y)$ and $A_{z}(Y)$, which is valid since $A_{z}$ is strictly monotone increasing.
} 


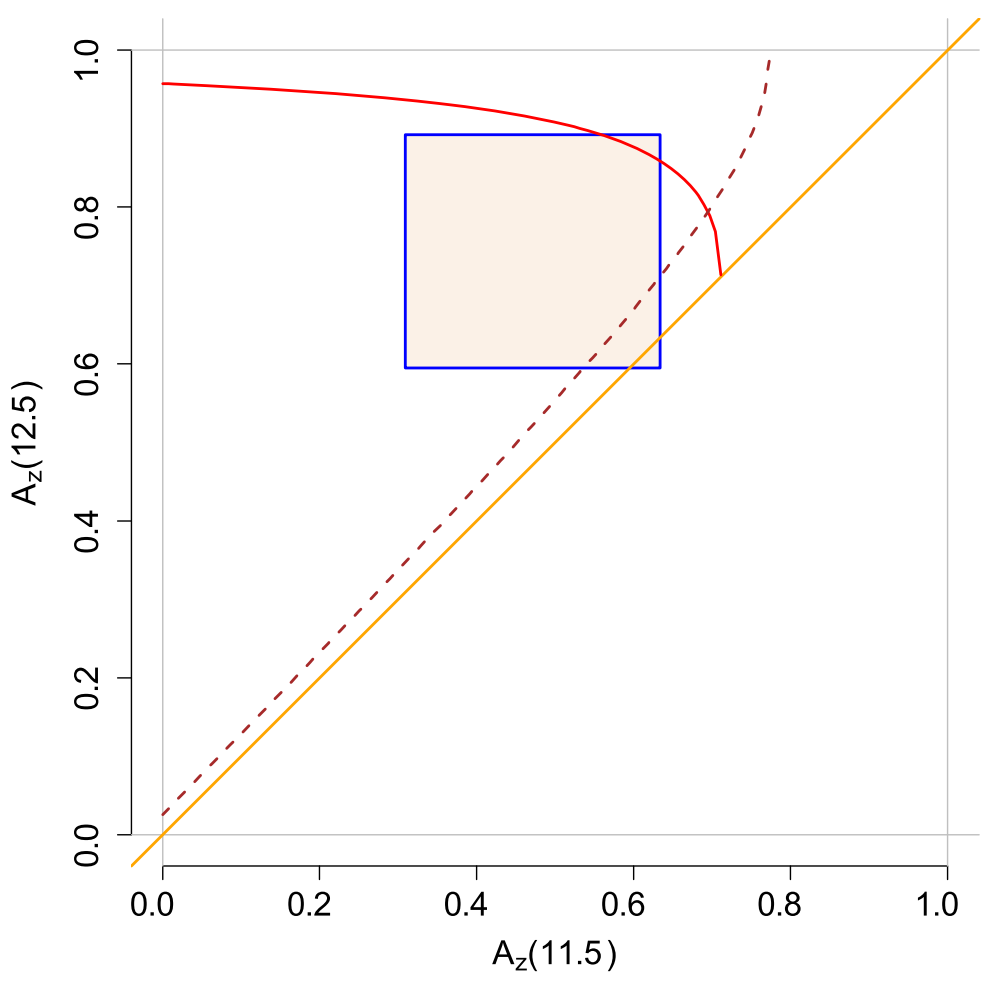

FIGURE 1.- Values of valuation distribution functions $\phi=\left(A_{z}(11.5), A_{z}(12.5)\right)$ for a particular distribution of ordered final bids. The straight light solid line is the $45^{\circ}$ line. The rectangular box shows the HT pointwise bounds. The solid curved and dashed lines are delivered by the new inequalities. Values of $\phi$ above the solid curved line, below the dashed line, or outside the HT box cannot lie on valuation distribution functions in the identified set.

bid of a low valuation bidder is their valuation minus an amount that is the absolute value of an independent realization of a standard normal variable. ${ }^{27}$ The final bid of a high valuation bidder is a weighted average of the low and high valuations with the weight given by a realization of a uniform variate with support on $[0,1]$. For the purpose of illustration, containment functional probabilities (6.6) are approximated using the result of $10^{6}$ simulated independent auctions from this population.

Figure 1 shows combinations of values of the valuation distribution function $A_{z}(v)$ at $v \in(11.5,12.5)$ with $A_{z}(11.5)$ plotted horizontally. The boundaries of the rectangular region in Figure 1 shows the upper and lower HT pointwise bounds on $\phi \equiv$ ( $\left.A_{z}(11.5), A_{z}(12.5)\right)$ obtained with the distribution of ordered final bids just described. The valuation distribution function is monotone increasing so the HT pointwise bounds admit only values of $\phi$ inside the rectangular region and above the $45^{\circ}$ line. The curved lines show the bounds delivered using $(6.8)$ with $\left(v_{1}, v_{2}, v_{3}\right)=(11.5,12.5, \infty)$ (solid) and $\left(v_{1}, v_{2}, v_{3}\right)=(11.5,11.5,12.5)$ (dashed). Only values of $\phi$ lying below the solid curve and above the dashed curve and in the rectangular box are feasible values of valuation distribution functions that reside in the identified set. There are similar results at other choices

\footnotetext{
${ }^{27}$ Zero in the unlikely event this calculation delivers a negative number.
} 
of values of $V$. The impact of the new restrictions on the identified set will depend on the distribution of ordered final bids.

Considering all possible choices for $y^{\prime}$ and $y_{M}^{\prime \prime}$ in the contiguous unions of $U$-level sets (6.3) yields, via (6.5), a dense system of inequalities involving values of the conditional distribution function of valuations at all choices of up to $M+1$ values of its arguments. A complete characterization of the identified set of valuation distributions also involves consideration of an uncountable infinity of unions of contiguous unions so there is no limit to the number of coordinates of valuation distributions simultaneously constrained by the HT model.

This auction model example highlights two major advantages of the method we advance in this paper. First, our method can deliver the entire system of inequalities defining an identified set, many of which are not plain to see. Of course in computations with finite amounts of data and computing resources, a selection of inequalities must be made and research is needed on the design of such selections and, if design is data driven, on its impact on estimation and inference. Second, our method necessarily delivers a characterization of the sharp identified set, obviating the need for constructive proof of sharpness, which is difficult to obtain for the HT model and, indeed, for many other models.

\section{CONCLUSION}

This paper provides characterizations of identified sets of structures and structural features for a very broad class of models. It delivers results for complete and incomplete models and for partially and point identifying models. The results apply to models in which the inverse of the structural mapping from unobserved heterogeneity to observed endogenous variables may not be uniquely valued. Models with discrete and censored endogenous variables fall under this heading, as do models permitting general forms of multivariate unobserved heterogeneity, such as random coefficient models and models placing inequality constraints on unobserved and endogenous variables.

The results extend the scope of application of instrumental variables for use in structural econometric models, in view of which we have described the class of models covered here as generalized instrumental variable models. It is straightforward to incorporate instrumental variable restrictions involving conditional mean or quantile independence of unobserved variables and instruments as well as full stochastic independence. However, the coverage of the results is wider because in some of the models to which the results apply instrumental variable restrictions play no significant role. The incomplete model of English auctions studied in Section 6 is one such model.

The characterizations developed here always deliver sharp bounds, removing the need for case-by-case constructive proofs of sharpness. This is a great benefit since it is often difficult to formulate such proofs as, for example, is the case in the incomplete model of English auctions.

All of the characterizations of identified sets presented in this paper can be expressed as systems of conditional moment inequalities and equalities. These can be employed for estimation and inference using a variety of approaches from the recent literature. With discrete conditioning variables, identified sets can be expressed using unconditional moment inequalities, and inference may be conducted as in Chernozhukov, Hong, and Tamer (2007), Beresteanu and Molinari (2008), Romano and Shaikh (2008, 2010), Rosen (2008), Andrews and Guggenberger (2009), Andrews and Soares (2010), Andrews and Jia-Barwick (2010), Bugni (2010), Canay (2010), or Romano, Shaikh, and Wolf (2014). With continuous conditioning variables inference using conditional moment inequalities 
can be performed; see, for example, Andrews and Shi (2013, 2014), Chernozhukov, Lee, and Rosen (2013), Lee, Song, and Whang (2013b, 2013a), Armstrong (2014, 2015), and Chetverikov (2011).

In some models the number of inequality restrictions fully characterizing an identified set can be large relative to the sample size. This is a problem that arises more generally and it is the subject of current research; see, for example, Menzel (2009), Chernozhukov, Chetverikov, and Kato (2013), and Andrews and Shi (2017).

The complexity of the characterizations that can arise when using the results of this paper are simply the consequence of using complete characterizations of identified sets, which the methods of this paper always deliver. Compare, for example, the simplicity of the pointwise bounds on valuation distributions in the English auction model of Section 6 and the complexity of the complete characterization of the identified set of valuation distributions obtained using the results of this paper. The additional inequalities afforded by the sharp characterization will generally deliver tighter bounds, and so their use can be beneficial. In practice, the benefit of incorporating additional inequalities must be weighed against computational cost and the quality of inference must be considered too. In the working paper Chesher and Rosen (2015) we demonstrate approaches for selecting finite collections of inequalities from the uncountable infinity of inequalities characterizing the identified set in some continuous outcome models. There is a need for research into optimal inequality selection procedures that take into account the error in approximating the identified set when using a particular finite selection of inequalities and the quality of inference, which may decline as more inequalities are considered.

\section{APPENDIX: PROOFS}

Proof OF TheOREM 1: Fix $z \in \mathcal{R}_{Z}$ and suppose that $F_{Y \mid Z}(\cdot \mid z)$ is selectionable with respect to the conditional distribution of $\mathcal{Y}(U, Z ; h)$ given $Z=z$. By Restriction $\mathrm{A} 3, U$ is conditionally distributed $G_{U \mid Z}(\cdot \mid z)$ given $Z=z$, and thus selectionability implies that there exist random variables $\tilde{Y}$ and $\tilde{U}$ such that

(i) $\tilde{Y} \mid Z=z \sim F_{Y \mid Z}(\cdot \mid z)$,

(ii) $\tilde{U} \mid Z=z \sim G_{U \mid Z}(\cdot \mid z)$,

(iii) $\mathbb{P}[\tilde{Y} \in \mathcal{Y}(\tilde{U}, Z ; h) \mid Z=z]=1$.

By Restriction A3, $\tilde{Y} \in \mathcal{Y}(\tilde{U}, Z ; h)$ if and only if $h(\tilde{Y}, Z, \tilde{U})=0$; equivalently, $\tilde{U} \in$ $\mathcal{U}(\tilde{Y}, Z ; h)$. Condition (iii) is therefore equivalent to

$$
\mathbb{P}[\tilde{U} \in \mathcal{U}(\tilde{Y}, Z ; h) \mid Z=z]=1 .
$$

Thus there exist random variables $\tilde{Y}$ and $\tilde{U}$ satisfying (i) and (ii) such that (A.1) holds, and, equivalently, such that $G_{U \mid Z}(\cdot \mid z)$ is selectionable with respect to the conditional distribution of $\mathcal{U}(Y, Z ; h)$ given $Z=z$. The choice of $z$ was arbitrary, and the argument thus follows for all $z \in \mathcal{R}_{Z}$.

Q.E.D.

The proof of Theorem 2 follows directly from application of Theorem 1 to Definitions 2 and 3 .

PROOF OF COROLLARY 1: From the selectionability characterization of $\mathcal{M}^{*}$ in $U$-space in Theorem 2, we have that

$$
\mathcal{M}^{*}=\left\{\left(h, G_{U}\right) \in \mathcal{M}: G_{U}(\cdot \mid z) \preccurlyeq \mathcal{U}(Y, z ; h) \text { when } Y \sim F_{Y \mid Z}(\cdot \mid z) \text {, a.e. } z \in \mathcal{R}_{Z}\right\} \text {. }
$$


Fix $z \in \mathcal{R}_{Z}$ and suppose $Y \sim F_{Y \mid Z}(\cdot \mid z)$. From Artstein's inequality (see Artstein (1983), Norberg (1992), or Molchanov (2005, Section 1.4.8.)), $G_{U}(\cdot \mid z) \preccurlyeq \mathcal{U}(Y, z ; h)$ if and only if

$$
\forall \mathcal{K} \in \mathrm{K}\left(\mathcal{R}_{U}\right), \quad G_{U}(\mathcal{K} \mid z) \leq F_{Y \mid Z}[\mathcal{U}(Y, z ; h) \cap \mathcal{K} \neq \emptyset \mid z],
$$

where $\mathrm{K}\left(\mathcal{R}_{Z}\right)$ denotes the collection of all compact sets on $\mathcal{R}_{U}$. By Corollary 1.4.44 of Molchanov (2005) this is equivalent to

$$
\forall \mathcal{S} \in \mathrm{O}\left(\mathcal{R}_{U}\right), \quad G_{U}(\mathcal{S} \mid z) \leq F_{Y \mid Z}[\mathcal{U}(Y, z ; h) \cap \mathcal{S} \neq \emptyset \mid z],
$$

where $\mathrm{O}\left(\mathcal{R}_{U}\right)$ is the collection of all open subsets of $\mathcal{R}_{U}$. Because $G_{U}(\mathcal{S} \mid z)=1-$ $G_{U}\left(\mathcal{S}^{c} \mid z\right)$ and

$$
F_{Y \mid Z}\left[\mathcal{U}(Y, z ; h) \subseteq \mathcal{S}^{c} \mid z\right]=1-F_{Y \mid Z}[\mathcal{U}(Y, z ; h) \cap \mathcal{S} \neq \emptyset \mid z],
$$

this is equivalent to

$$
\forall \mathcal{S} \in \mathrm{O}\left(\mathcal{R}_{U}\right), \quad F_{Y \mid Z}\left[\mathcal{U}(Y, z ; h) \subseteq \mathcal{S}^{c} \mid z\right] \leq G_{U}\left(\mathcal{S}^{c} \mid z\right) .
$$

The collection of $\mathcal{S}^{c}$ such that $\mathcal{S} \in \mathrm{O}\left(\mathcal{R}_{U}\right)$ is precisely the collection of closed sets on $\mathcal{R}_{U}$, $\mathrm{F}\left(\mathcal{R}_{U}\right)$, completing the proof.

Q.E.D.

Proof of LemMa 1: The set $\mathcal{U}_{\mathcal{S}}(h, z)$ is a union of sets contained in $\mathcal{S}$, so that $\mathcal{U}_{\mathcal{S}}(h, z) \subseteq \mathcal{S}$ and

$$
G_{U \mid Z}\left(\mathcal{U}_{\mathcal{S}}(h, z) \mid z\right) \leq G_{U \mid Z}(\mathcal{S} \mid z) .
$$

By supposition we have

$$
C_{h}\left(\mathcal{U}_{\mathcal{S}}(h, z) \mid z\right) \leq G_{U \mid Z}\left(\mathcal{U}_{\mathcal{S}}(h, z) \mid z\right) .
$$

The result then holds because $C_{h}(\mathcal{S} \mid z)=C_{h}\left(\mathcal{U}_{\mathcal{S}}(h, z) \mid z\right)$, since

$$
\begin{aligned}
C_{h}\left(\mathcal{U}_{\mathcal{S}}(h, z) \mid z\right) & \equiv \mathbb{P}\left[\mathcal{U}(Y, Z ; h) \subseteq \mathcal{U}_{\mathcal{S}}(h, z) \mid Z=z\right] \\
& =\int_{y \in \mathcal{R}_{Y \mid z}} 1\left[\mathcal{U}(y, z ; h) \subseteq \mathcal{U}_{\mathcal{S}}(h, z)\right] d F_{Y \mid Z}^{*}(y \mid z) \\
& =\int_{y \in \mathcal{R}_{Y \mid z}} 1[\mathcal{U}(y, z ; h) \subseteq \mathcal{S}] d F_{Y \mid Z}^{*}(y \mid z) \\
& =C_{h}(\mathcal{S} \mid z),
\end{aligned}
$$

where $F_{Y \mid Z}^{*}$ denotes the conditional cumulative distribution function of $Y$ given $Z$. The second line follows by the law of total probability, and the third line follows by definition of $\mathcal{U}_{\mathcal{S}}(h, z)$ in (4.2). Combining $C_{h}\left(\mathcal{U}_{\mathcal{S}}(h, z) \mid z\right)=C_{h}(\mathcal{S} \mid z)$ with (A.2) and (A.3) completes the proof.

Q.E.D.

Proof of Theorem 3: Fix $(h, z)$. Suppose that

$$
\forall \mathcal{U} \in \mathrm{Q}(h, z), \quad C_{h}(\mathcal{U} \mid z) \leq G_{U \mid Z}(\mathcal{U} \mid z) .
$$


Let $\mathcal{S} \in \mathrm{U}^{*}(h, z)$ and $\mathcal{S} \notin \mathrm{Q}(h, z)$. Since $\mathcal{S} \notin \mathrm{Q}(h, z)$ there exist nonempty collections of sets $\mathrm{S}_{1}, \mathrm{~S}_{2} \in \mathrm{U}^{\mathcal{S}}(h, z)$ with $\mathrm{S}_{1} \cup \mathrm{S}_{2}=\mathrm{U}^{\mathcal{S}}(h, z)$ such that

$$
\mathcal{S}_{1} \equiv \bigcup_{\mathcal{T} \in \mathrm{S}_{1}} \mathcal{T} \in \mathrm{Q}(h, z), \quad \mathcal{S}_{2} \equiv \bigcup_{\mathcal{T} \in \mathrm{S}_{2}} \mathcal{T} \in \mathrm{Q}(h, z)
$$

and

$$
G_{U \mid Z}\left(\mathcal{S}_{1} \cap \mathcal{S}_{2} \mid z\right)=0 .
$$

Since $\mathcal{S}_{1}, \mathcal{S}_{2} \in \mathrm{Q}(h, z)$ we also have that

$$
C_{h}\left(\mathcal{S}_{1} \mid z\right) \leq G_{U \mid Z}\left(\mathcal{S}_{1} \mid z\right) \quad \text { and } \quad C_{h}\left(\mathcal{S}_{2} \mid z\right) \leq G_{U \mid Z}\left(\mathcal{S}_{2} \mid z\right)
$$

Because $\mathrm{S}_{1} \cup \mathrm{S}_{2}=\mathrm{U}^{\mathcal{S}}(h, z)$,

$$
\mathcal{U}(Y, z ; h) \subseteq \mathcal{S} \Rightarrow\left\{\mathcal{U}(Y, z ; h) \subseteq \mathcal{S}_{1} \text { or } \mathcal{U}(Y, z ; h) \subseteq \mathcal{S}_{2}\right\}
$$

Using (A.7), (A.6), and (A.5) in sequence we then have

$$
C_{h}(\mathcal{S} \mid z) \leq C_{h}\left(\mathcal{S}_{1} \mid z\right)+C_{h}\left(\mathcal{S}_{2} \mid z\right) \leq G_{U \mid Z}\left(\mathcal{S}_{1} \mid z\right)+G_{U \mid Z}\left(\mathcal{S}_{2} \mid z\right)=G_{U \mid Z}(\mathcal{S} \mid z) .
$$

Combined with (A.4) this implies $C_{h}(\mathcal{S} \mid z) \leq G_{U \mid Z}(\mathcal{S} \mid z)$ for all $\mathcal{S} \in \mathrm{U}^{*}(h, z)$ and hence all $\mathcal{S} \subseteq \mathcal{R}_{U}$ by Lemma 1 , completing the proof.

Q.E.D.

Proof of Corollary 2: Consider any $\mathcal{S} \in \mathrm{Q}^{E}(h, z)$. Then for all $y \in \mathcal{Y}$, either $\mathcal{U}(y, z ; h) \subseteq \mathcal{S}$ or $\mathcal{U}(y, z ; h) \subseteq \operatorname{cl}\left(\mathcal{S}^{c}\right)$. Thus

$$
C_{h}(\mathcal{S} \mid z)+C_{h}\left(\operatorname{cl}\left(\mathcal{S}^{c}\right) \mid z\right)=\mathbb{P}[\mathcal{U}(Y, Z ; h) \subseteq \mathcal{S} \mid z]+\mathbb{P}\left[\mathcal{U}(Y, Z ; h) \subseteq \operatorname{cl}\left(\mathcal{S}^{c}\right) \mid z\right]=1 .
$$

The inequalities of Theorem 3 imply that

$$
G_{U \mid Z}(\mathcal{S} \mid z) \geq C_{h}(\mathcal{S} \mid z) \quad \text { and } \quad G_{U \mid Z}\left(\operatorname{cl}\left(\mathcal{S}^{c}\right) \mid z\right) \geq C_{h}\left(\operatorname{cl}\left(\mathcal{S}^{c}\right) \mid z\right)
$$

Then $G_{U \mid Z}(\partial \mathcal{S} \mid z)=0$ implies that $G_{U \mid Z}(\mathcal{S} \mid z)+G_{U \mid Z}\left(\operatorname{cl}\left(\mathcal{S}^{c}\right) \mid z\right)=1$, which taken with (A.8) implies that both inequalities hold with equality.

Q.E.D.

ProOF OF THEOREM 4: By Restriction SI, $G_{U \mid Z}(\cdot \mid z)=G_{U}(\cdot)$ a.e. $z \in \mathcal{R}_{Z}$. Equations (5.3) and (5.1) follow from (3.1) and Theorem 2, respectively, upon substituting $G_{U}(\cdot)$ for $G_{U \mid Z}(\cdot \mid z)$. Equation (5.2) follows by Corollary 2, again replacing $G_{U \mid Z}(\cdot \mid z)$ with $G_{U}(\cdot)$. Equivalence of (5.1) and (5.4) with $G_{U \mid Z}(\cdot \mid z)=G_{U}(\cdot)$ holds by Artstein's inequality; see, for example, Molchanov (2005, Corollary 4.44, pp. 69-70).

Q.E.D.

ProOF OF COROLlary 3: For any $\left(h, G_{U}\right) \in \mathcal{M}^{*}$ we have that for any $\mathcal{S}$ and a.e. $z \in$ $\mathcal{R}_{Z}$, both $C_{h}\left(\mathcal{S}^{c} \mid z\right) \leq G_{U}\left(\mathcal{S}^{c}\right)$ and $C_{h}(\mathcal{S} \mid z) \leq G_{U}(\mathcal{S})$. Then since $G_{U}\left(\mathcal{S}^{c}\right)=1-G_{U}(\mathcal{S})$,

$$
C_{h}(\mathcal{S} \mid z) \leq G_{U}(\mathcal{S})=1-G_{U}\left(\mathcal{S}^{c}\right) \leq 1-C_{h}\left(\mathcal{S}^{c} \mid z\right)
$$

If in addition $G_{U}(\partial \mathcal{S})=0$, then $G_{U}\left(\mathcal{S}^{c}\right)=G_{U}\left(\operatorname{cl}\left(\mathcal{S}^{c}\right)\right)$ and $G_{U}(\mathcal{S})=G_{U}(\operatorname{cl}(\mathcal{S}))$. Since $C_{h}\left(\operatorname{cl}\left(\mathcal{S}^{c}\right) \mid z\right) \leq G_{U}\left(\operatorname{cl}\left(\mathcal{S}^{c}\right)\right)$, then

$$
C_{h}(\operatorname{cl}(\mathcal{S}) \mid z) \leq G_{U}(\operatorname{cl}(\mathcal{S}))=G_{U}(\mathcal{S})=1-G_{U}\left(\operatorname{cl}\left(\mathcal{S}^{c}\right)\right) \leq 1-C_{h}\left(\operatorname{cl}\left(\mathcal{S}^{c}\right) \mid z\right)
$$

Since (A.9) and (A.10) hold for a.e. $z \in \mathcal{R}_{Z}$, this completes the proof.

Q.E.D. 
ProOF OF THEOREM 5: Restrictions A3 and A5 guarantee that $\mathcal{U}(Y, Z ; h)$ is integrable and closed. In particular integrability holds because by Restriction A3 first $G_{U \mid Z}(\mathcal{S} \mid z) \equiv \mathbb{P}[U \in \mathcal{S} \mid z]$ so that, for some finite $c \in \mathcal{C}, E[U \mid z]=c$ a.e. $z \in \mathcal{R}_{Z}$, and second $\mathbb{P}[h(Y, Z, U)=0]=1$ so that

$$
U \in \mathcal{U}(Y, Z ; h) \equiv\left\{u \in \mathcal{R}_{U}: h(Y, Z, u)=0\right\},
$$

implying that $\mathcal{U}(Y, Z ; h)$ has an integrable selection, namely $U$. From Definition $6, c \in$ $\mathbb{E}[\mathcal{U}(Y, Z ; h) \mid z]$ a.e. $z \in \mathcal{R}_{Z}$ therefore holds if and only if there exists a random variable $\tilde{U} \in \operatorname{Sel}(\mathcal{U}(Y, Z ; h))$ such that $E[\tilde{U} \mid z]=c$ a.e. $z \in \mathcal{R}_{Z}$, and hence $\mathcal{H}^{*}$ is the identified set for $h$. The representation of the identified set of structures $\mathcal{M}^{*}$ then follows directly from Theorem 2 .

Q.E.D.

ProOF OF COROLlARY 4: Restrictions A3 and A5 and the continuity requirement of Restriction $\mathrm{MI}^{*}$ guarantee that $\mathcal{D}(Y, Z ; h)$ is integrable and closed. From Definition 6 , for any $c \in \mathcal{C}, c \in \mathbb{E}[\mathcal{D}(Y, Z ; h) \mid z]$ a.e. $z \in \mathcal{R}_{Z}$ therefore holds if and only if there exists a random variable $D \preccurlyeq \mathcal{D}(Y, Z ; h)$ such that $E[D \mid z]=c$ a.e. $z \in \mathcal{R}_{Z}$. The condition $D \preccurlyeq$ $\mathcal{D}(Y, Z ; h)$ ensures that

$$
\mathbb{P}[D \in \mathcal{D}(Y, Z ; h) \mid z]=1, \quad \text { a.e. } z \in \mathcal{R}_{Z} .
$$

Define

$$
\widetilde{\mathcal{U}}(D, Y, Z ; h) \equiv\{u \in \mathcal{U}(Y, Z ; h): D=d(u, Z)\} .
$$

By the definition of $\mathcal{D}(Y, Z ; h), D \in \mathcal{D}(Y, Z ; h)$ implies that $\tilde{\mathcal{U}}(D, Y, Z ; h)$ is nonempty. Hence there exists a random variable $\tilde{U}$ such that with probability $1, \tilde{U} \in \tilde{\mathcal{U}}(D, Y, Z ; h) \subseteq$ $\mathcal{U}(Y, Z ; h)$, where $D=d(\tilde{U}, Z)$. Thus $\tilde{U}$ is a measurable selection of $\mathcal{U}(Y, Z ; h)$ and $E[d(\tilde{U}, Z) \mid z]=c$ a.e. $z \in \mathcal{R}_{Z}$. Therefore $\mathcal{H}^{*}$ is the identified set for $h$, and the given characterization of $\mathcal{M}^{*}$ follows.

Q.E.D.

Proof of Theorem 6: Using Corollary 1 and Definition 4 with $\psi\left(h, \mathcal{G}_{U \mid Z}\right)=h$, the identified set of structural functions $h$ is

$$
\mathcal{H}^{* *}=\left\{h \in \mathcal{H}: \exists \mathcal{G}_{U \mid Z} \in \mathrm{G}_{U \mid Z} \text { s.t. } \forall \mathcal{S} \in \mathrm{F}\left(\mathcal{R}_{U}\right), C_{h}(\mathcal{S} \mid z) \leq G_{U \mid Z}(\mathcal{S} \mid z) \text { a.e. } z \in \mathcal{R}_{Z}\right\}
$$

We begin by considering claim (i) of the theorem. Consider any $h \in \mathcal{H}^{* *}$. We first seek to show that $h \in \mathcal{H}^{*}$. Since $h \in \mathcal{H}^{* *}$ there exists $c \in C$ and $\mathcal{G}_{U \mid Z}$ such that $q_{U \mid Z}(\tau \mid z)=c$ a.e. $z \in \mathcal{R}_{Z}$. Fix $z \in \mathcal{R}_{Z}$. Then from $C_{h}(\mathcal{S} \mid z) \leq G_{U \mid Z}(\mathcal{S} \mid z)$ in $($ A.11 $),{ }^{28}$

$$
C_{h}((-\infty, c) \mid z) \leq G_{U \mid Z}((-\infty, c) \mid z) \leq \tau,
$$

where the second inequality holds because $q_{U \mid Z}(\tau \mid z)=c$, and because of Restriction IS, $\mathcal{U}(Y, Z ; h)=[\underline{u}(Y, Z ; h), \bar{u}(Y, Z ; h)]$,

$$
C_{h}((-\infty, c) \mid z)=\mathbb{P}[\bar{u}(Y, Z ; h)<c \mid z] .
$$

Similarly, for $\mathcal{S}=(c, \infty)$ it follows that

$$
C_{h}((c, \infty) \mid z) \leq G_{U \mid Z}((c, \infty) \mid z) \leq 1-\tau,
$$

\footnotetext{
${ }^{28}$ Note that if $C_{h}(\mathcal{S} \mid z) \leq G_{U \mid Z}(\mathcal{S} \mid z)$ holds for all closed $\mathcal{S}$, then it also holds for all open $\mathcal{S}$ by the same reasoning as in Corollary 1 .
} 
and again using Restriction IS,

$$
C_{h}((c, \infty) \mid z)=1-\mathbb{P}[\underline{u}(Y, Z ; h) \leq c \mid z] .
$$

Combining this with (A.14) and also using (A.12) and (A.13) above gives

$$
\mathbb{P}[\bar{u}(Y, Z ; h)<c \mid z] \leq \tau \leq \mathbb{P}[\underline{u}(Y, Z ; h) \leq c \mid z] .
$$

The choice of $z$ was arbitrary, so the above holds a.e. $z \in \mathcal{R}_{Z}$, implying that $h \in \mathcal{H}^{*}$.

Now consider any $h \in \mathcal{H}^{*}$. We wish to show that $h \in \mathcal{H}^{* *}$. It suffices to show that for any such $h$ under consideration there exists a collection of conditional distributions $\mathcal{G}_{U \mid Z}$ such that for almost every $z \in \mathcal{R}_{Z}$, (a) $G_{U \mid Z}(\cdot \mid z)$ has $\tau$-quantile equal to $c$, and (b) $\forall \mathcal{S} \in \mathrm{F}\left(\mathcal{R}_{U}\right)$, $C_{h}(\mathcal{S} \mid z) \leq G_{U \mid Z}(\mathcal{S} \mid z)$.

To do so we fix an arbitrary $z \in \mathcal{R}_{Z}$ and verify the existence of a random variable $\tilde{U}$ distributed $G_{U \mid Z}(\cdot \mid z)$ such that (a) and (b) hold. One such is

$$
\tilde{U} \equiv D \cdot \underline{u}(Y, Z ; h)+(1-D) \cdot \bar{u}(Y, Z ; h),
$$

where $D$ is a Bernoulli random variable defined on $(\Omega, L, \mathbb{P})$ with parameter $\lambda(z)=$ $\mathbb{P}[D=1 \mid z]$, which is independent of $(Y, Z)$, and where $\lambda(z)$ solves

$$
\lambda(z)(\mathbb{P}[\underline{u}(Y, Z ; h) \leq c \mid z]-\mathbb{P}[\bar{u}(Y, Z ; h)<c \mid z])=\tau-\mathbb{P}[\bar{u}(Y, Z ; h)<c \mid z] .
$$

It follows that $\mathbb{P}[\tilde{U} \leq c \mid z]=\tau$ so that (a) holds. ${ }^{29}$ To verify (b) note that for any $\mathcal{S} \in \mathrm{F}\left(\mathcal{R}_{U}\right)$,

$$
G_{U \mid Z}(\mathcal{S} \mid z)=\mathbb{P}[\tilde{U} \in \mathcal{S} \mid z] \geq \mathbb{P}[[\underline{u}(Y, Z ; h), \bar{u}(Y, Z ; h)] \subseteq \mathcal{S} \mid z]=C_{h}(\mathcal{S} \mid z),
$$

where the inequality holds because

$$
[\underline{u}(Y, Z ; h), \bar{u}(Y, Z ; h)] \subseteq \mathcal{S} \Rightarrow \tilde{U} \in \mathcal{S} .
$$

Thus (b) holds, and since the choice $z$ was arbitrary, $h \in \mathcal{H}^{* *}$ as desired. This verifies claim (i).

Claim (ii) holds because with $\bar{u}(Y, Z ; h)$ and $\underline{u}(Y, Z ; h)$ continuously distributed given $Z=z$, a.e. $z \in \mathcal{R}_{Z}$, their conditional cumulative distribution functions are invertible at $c$ and the lower bound in (A.16) is equal to $\mathbb{P}[\bar{u}(Y, Z ; h) \leq c \mid z]$. Thus for any $z \in \mathcal{R}_{Z}$,

$$
\underline{q}(\tau, z ; h) \leq c \leq \bar{q}(\tau, z ; h) \quad \Leftrightarrow \quad \mathbb{P}[\bar{u}(Y, Z ; h) \leq c \mid z] \leq \tau \leq \mathbb{P}[\underline{u}(Y, Z ; h) \leq c \mid z] .
$$

Claim (iii) follows directly from Theorem 2.

Q.E.D.

\section{REFERENCES}

ANDREws, D. W. K., AND P. GugGenBerger (2009): "Validity of Subsampling and Plug-in Asymptotic Inference for Parameters Defined by Moment Inequalities," Econometric Theory, 25 (3), 669-709. [982]

ANDREWS, D. W. K., AND P. JIA-BARWICK (2010): "Inference for Parameters Defined by Moment Inequalities: A Recommended Moment Selection Procedure," Econometrica, 80 (6), 2805-2826. [982]

ANDREWS, D. W. K., AND X. SHI (2013): "Inference Based on Conditional Moment Inequalities," Econometrica, 81 (2), 609-666. [983]

${ }^{29}$ When $\mathbb{P}[\underline{u}(Y, Z ; h) \leq c \mid z]=\mathbb{P}[\bar{u}(Y, Z ; h)<c \mid z]$ the value of $\lambda(z)$ is irrelevant because then, in view of (A.17), $\mathbb{P}[\tilde{U} \leq c \mid z]=\tau$ for any $\lambda(z) \in[0,1]$. 
(2014): "Nonparametric Inference Based on Conditional Moment Inequalities," Journal of Econometrics, 179 (1), 31-45. [983]

(2017): "Inference Based on Many Conditional Moment Inequalities," Journal of Econometrics, 196 (2), 275-287. [983]

Andrews, D. W. K., AND G. SoARes (2010): "Inference for Parameters Defined by Moment Inequalities Using Generalized Moment Selection,” Econometrica, 78 (1), 119-157. [982]

ARADILlas-LOPEZ, A., AND A. M. Rosen (2013): "Inference in Ordered Response Games With Complete Information," CEMMAP Working Paper CWP33/13. [961]

ARMSTRONG, T. B. (2014): "Weighted KS Statistics for Inference on Conditional Moment Inequalities," Journal of Econometrics, 181 (2), 92-116. [983]

(2015): "Asymptotically Exact Inference in Conditional Moment Inequality Models," Journal of Econometrics, 186 (1), 51-65. [983]

ARTSTEIN, Z. (1983): "Distributions of Random Sets and Random Selections," Israel Journal of Mathematics, 46 (4), 313-324. [962,969,984]

BASMANN, R. L. (1959): "A Generalized Classical Method of Linear Estimation of Coefficients in a Structural Equation," Econometrica, 25 (1), 77-83. [960]

Beresteanu, A., AND F. MOLINARI (2008): "Asymptotic Properties for a Class of Partially Identified Models," Econometrica, 76 (4), 763-814. [982]

Beresteanu, A., I. Molchanov, AND F. Molinari (2011): "Sharp Identification Regions in Models With Convex Moment Predictions," Econometrica, 79 (6), 1785-1821. [961]

BowDEN, R. (1973): “The Theory of Parametric Identification,” Econometrica, 41 (6), 1069-1074. [962,967]

Bugni, F. (2010): "Bootstrap Inference for Partially Identified Models Defined by Moment Inequalities: Coverage of the Identified Set," Econometrica, 78 (2), 735-753. [982]

CANAY, I. (2010): "EL Inference for Partially Identified Models: Large Deviations Optimality and Bootstrap Validity," Journal of Econometrics, 156 (2), 408-425. [982]

Chernozhukov, V., AND C. HANSEN (2005): "An IV Model of Quantile Treatment Effects," Econometrica, 73 (1), 245-261. [960,971]

Chernozhukov, V., D. Chetverikov, AND K. Kato (2013): “Testing Many Moment Inequalities," CeMMAP Working Paper CWP65/13. [983]

Chernozhukov, V., H. Hong, AND E. TAmer (2007): "Estimation and Confidence Regions for Parameter Sets in Econometric Models," Econometrica, 75 (5), 1243-1284. [982]

Chernozhukov, V., S. LeE, AND A. M. Rosen (2013): "Intersection Bounds: Estimation and Inference," Econometrica, 81 (2), 667-737. [983]

CHESHER, A. (2010): "Instrumental Variable Models for Discrete Outcomes," Econometrica, 78 (2), 575-601. $[961,966]$

[2013): "Semiparametric Structural Models of Binary Response: Shape Restrictions and Partial Identification," Econometric Theory, 29 (2), 231-266. [961]

Chesher, A., AND A. M. Rosen (2012): "Simultaneous Equations Models for Discrete Outcomes: Coherence, Completeness, and Identification," CeMMAP Working Paper CWP21/12. [960,961,967,971]

(2013): "What Do Instrumental Variable Models Deliver With Discrete Dependent Variables?" American Economic Review: Papers and Proceedings, 103 (3), 557-562. [961,966]

(2015): "Characterizations of Identified Sets Delivered by Structural Econometric Models," CeMMAP Working Paper CWP63/15. [967,971,973,977,983]

CHESHER, A., AND K. SMOLINSKI (2012): "IV Models of Ordered Choice," Journal of Econometrics, 166 (1), 33-48. [966]

CHESher, A., A. M. Rosen, AND K. SMOLInSKi (2013): "An Instrumental Variable Model of Multiple Discrete Choice," Quantitative Economics, 4 (2), 157-196. [961,966]

Chetverikov, D. (2011): “Adaptive Test of Conditional Moment Inequalities," CeMMAP Working Paper CWP36/12. [983]

DAVID, H. A., AND H. N. NAGARAJA (2003): Order Statistics (Third Ed.). Hoboken: Wiley. [979]

GAlichon, A., AND M. Henry (2011): "Set Identification in Models With Multiple Equilibria," Review of Economic Studies, 78 (4), 1264-1298. [961,962,970,972,973]

HAILE, P. A., AND E. TAMER (2003): "Inference With an Incomplete Model of English Auctions," Journal of Political Economy, 111 (1), 1-51. [959,961,962,965,978]

Henry, M., R. MEANGo, AND M. Queyranne (2015): "Combinatorial Approach to Inference in Partially Identified Incomplete Structural Models,” Quantitative Economics, 6 (2), 499-529. [973]

HonG, H., AND E. TAMER (2003): "Inference in Censored Models With Endogenous Regressors," Econometrica, 71 (3), 905-932. [977] 
Hurwicz, L. (1950): "Generalization of the Concept of Identification," in Statistical Inference in Dynamic Economic Models. Research in Economics, ed. by T. C. Koopmans. New York: Wiley, 245-257. [962,967]

Khan, S., M. Ponomareva, AND E. TAMER (2011): "Sharpness in Randomly Censored Linear Models," Economics Letters, 113 (1), 23-25. [977]

Koopmans, T. (1949): "Identification Problems in Economic Model Construction," Econometrica, 17 (125144), 2. [962]

KOOPMANS, T., AND O. REIERSøL (1950): “The Identification of Structural Characteristics," Annals of Mathematical Statistics, 21 (165-181), 1-27. [962,967]

LANG, R. (1986): "A Note on the Measurability of Convex Sets," Arch. Math., 47 (1), 90-92. [974]

LEE, S., K. SONG, AND Y.-J. WHANG (2013a): “Testing for a General Class of Functional Inequalities,” Working Paper, Seoul National University. [983]

(2013b): “Testing Functional Inequalities,” Journal of Econometrics, 172 (1), 14-32. [983]

MANSKI, C. F., AND E. TAMER (2002): "Inference on Regressions With Interval Data on a Regressor or Outcome," Econometrica, 70 (2), 519-546. [966]

MASTEN, M. (2014): "Random Coefficients on Endogenous Variables in Simultaneous Equations Models," CeMMAP Working Paper CWP01/14. [966]

MATZKIN, R. (2007): "Nonparametric Identification," in The Handbook of Econometrics, Vol. 6b, ed. by J. J. Heckman and E. E. Leamer. Amsterdam: North-Holland, 5307-5368. [962,967]

(2008): "Identification in Nonparametric Simultaneous Equations Models," Econometrica, 76 (5), 945-978. [962,967]

MenZEL, K. (2009): "Consistent Estimation With Many Moment Inequalities," Journal of Econometrics, 182 (2), 329-350. [983]

MolCHANOV, I. S. (2005): Theory of Random Sets. London: Springer. [961,964,968,969,974,975,984,985]

NEWEY, W. K., AND J. L. POWELL (1989): “Nonparametric Instrumental Variable Estimation,” Working Paper, Princeton University. [960]

(2003): "Instrumental Variable Estimation of Nonparametric Models," Econometrica, 71 (5), 15651578. $[960,971]$

NGUYen, H. T. (2006): An Introduction to Random Sets. Boca Raton: Chapman and Hall. [974]

NorberG, T. (1992): "On the Existence of Ordered Couplings of Random Sets-With Applications," Israel Journal of Mathematics, 77 (3), 241-264. [969,984]

ROMANO, J., AND A. SHAIKH (2008): "Inference for Identifiable Parameters in Partially Identified Econometric Models," Journal of Planning and Statistical Inference, 138 (9), 2786-2807. [982]

Romano, J., A. SHAIKH, AND M. WOLF (2014): "A Practical Two-Step Method for Testing Moment Inequalities With an Application to Inference in Partially Identified Models," Econometrica, 82 (5), 1979-2002. [982]

ROMANO, J. P., AND A. M. SHAIKH (2010): "Inference for the Identified Set in Partially Identified Econometric Models," Econometrica, 78 (1), 169-211. [982]

Rosen, A. M. (2008): "Confidence Sets for Partially Identified Parameters That Satisfy a Finite Number of Moment Inequalities," Journal of Econometrics, 146 (1), 107-117. [982]

(2012): "Set Identification via Quantile Restrictions in Short Panels," Journal of Econometrics, 166 (1), 127-137. [961]

RotHenberG, T. J. (1971): "Identification in Parametric Models," Econometrica, 39 (3), 577-591. [962,967]

SuTHERLAND, W. A. (2009): Introduction to Metric and Topological Spaces. New York: Oxford Univerity Press. [964]

THEIL, H. (1953): Repeated Least Squares Applied to Complete Equation Systems. The Hague: Central Planning Bureau. [960]

WRight, P. (1928): The Tariff on Animal and Vegetable Oils. New York: Macmillan. [960]

Dept. of Economics, University College London, Gower Street, London, WC1E 6BT, U.K.; andrew.chesher@ucl.ac.uk

$$
\text { and }
$$

Dept. of Economics, Duke University, 213 Social Sciences, Box 90097, Durham, NC 27708, U.S.A.; adam.rosen@duke.edu.

Co-editor Elie Tamer handled this manuscript.

Manuscript received 17 January, 2014; final version accepted 20 October, 2016; available online 2 November, 2016. 